\title{
Spectroscopy of High-Redshift Supernovae from the ESSENCE Project: The First 2 Years
}

\section{Citation}

Matheson, Thomas, Stéphane Blondin, Ryan J. Foley, Ryan Chornock, Alexei V. Filippenko, Bruno Leibundgut, R. Chris Smith, et al. 2005. "Spectroscopy of High-Redshift Supernovae from the ESSENCE Project: The First 2 Years." The Astronomical Journal 129 (5): 2352-75. https:// doi.org/10.1086/429679.

\section{Permanent link}

http://nrs.harvard.edu/urn-3:HUL.InstRepos:41417344

\section{Terms of Use}

This article was downloaded from Harvard University's DASH repository, and is made available under the terms and conditions applicable to Other Posted Material, as set forth at http:// nrs.harvard.edu/urn-3:HUL.InstRepos:dash.current.terms-of-use\#LAA

\section{Share Your Story}

The Harvard community has made this article openly available. Please share how this access benefits you. Submit a story.

Accessibility 


\title{
SPECTROSCOPY OF HIGH-REDSHIFT SUPERNOVAE FROM THE ESSENCE PROJECT: THE FIRST 2 YEARS $^{1}$
}

\author{
Thomas Matheson, ${ }^{2}$ Stéphane Blondin, ${ }^{3}$ Ryan J. Foley, ${ }^{4}$ Ryan Chornock, ${ }^{4}$ Alexei V. Filippenko, ${ }^{4}$ Bruno Leibundgut, ${ }^{3}$

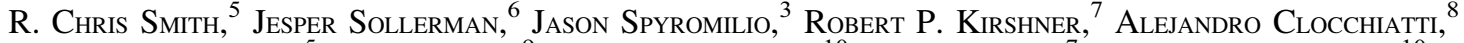 \\ Claudio Aguilera, ${ }^{5}$ Brian Barris, ${ }^{9}$ Andrew C. Becker, ${ }^{10}$ Peter Challis, ${ }^{7}$ Ricardo Covarrubias, ${ }^{10}$ \\ Peter Garnavich, ${ }_{11}^{11}$ Malcolm Hicken, ${ }^{7,12}$ Saurabh Jha, ${ }^{4}$ Kevin Krisciunas, ${ }^{11}$ Weidong Li, ${ }^{4}$ \\ Anthony Miceli, ${ }^{10}$ Gajus Miknaitis, ${ }^{13}$ Jose Luis Prieto, ${ }^{14}$ Armin Rest, ${ }^{5}$ Adam G. Riess, ${ }^{15}$ \\ Maria Elena Salvo, ${ }^{16}$ Brian P. Schmidt, ${ }^{16}$ Christopher W. Stubbs, ${ }^{7,12}$ \\ Nicholas B. SuntzefF, ${ }^{5}$ and John L. Tonry 9 \\ Received 2004 November 10; accepted 2005 February 13
}

\begin{abstract}
We present the results of spectroscopic observations of targets discovered during the first 2 years of the ESSENCE project. The goal of ESSENCE is to use a sample of 200 Type Ia supernovae (SNe Ia) at moderate redshifts $(0.2 \lesssim z \lesssim 0.8)$ to place constraints on the equation of state of the universe. Spectroscopy not only provides the redshifts of the objects but also confirms that some of the discoveries are indeed SNe Ia. This confirmation is critical to the project, as techniques developed to determine luminosity distances to SNe Ia depend on the knowledge that the objects at high redshift have the same properties as the ones at low redshift. We describe the methods of target selection and prioritization, the telescopes and detectors, and the software used to identify objects. The redshifts deduced from spectral matching of high-redshift SNe Ia with low-redshift SNe Ia are consistent with those determined from host-galaxy spectra. We show that the high-redshift SNe Ia match well with low-redshift templates. We include all spectra obtained by the ESSENCE project, including 52 SNe Ia, five corecollapse SNe, 12 active galactic nuclei, 19 galaxies, four possibly variable stars, and 16 objects with uncertain identifications.
\end{abstract}

Key words: distance scale — galaxies: distances and redshifts - supernovae: general

\section{INTRODUCTION}

The revolution wrought in modern cosmology using luminosity distances of Type Ia supernovae (SNe Ia) (Schmidt et al. 1998; Riess et al. 1998, 2001, 2004b; Perlmutter et al. 1999; Knop et al. 2003; Tonry et al. 2003; Barris et al. 2004; see Filippenko 2004, 2005 for recent reviews) relies on the fact that

\footnotetext{
${ }^{1}$ Based in part on observations obtained at the Cerro Tololo Inter-American Observatory, which is operated by the Association of Universities for Research in Astronomy (AURA), Inc., under a cooperative agreement with the National Science Foundation (NSF); the European Southern Observatory, Chile (ESO Programme 170.A-0519); the Gemini Observatory, which is operated by AURA under a cooperative agreement with the NSF on behalf of the Gemini partnership (the NSF [United States], the Particle Physics and Astronomy Research Council [United Kingdom], the National Research Council [Canada], CONICYT [Chile], the Australian Research Council [Australia], CNPq [Brazil], and CONICET [Argentina] [programs GN-2002B-Q-14, GN-2003B-Q-14, and GS-2003B-Q-11]); the Magellan Telescopes at Las Campanas Observatory; the MMT Observatory, a joint facility of the Smithsonian Institution and the University of Arizona; and the F. L. Whipple Observatory, which is operated by the Smithsonian Astrophysical Observatory. Some of the data presented herein were obtained at the W. M. Keck Observatory, which is operated as a scientific partnership among the California Institute of Technology, the University of California, and the National Aeronautics and Space Administration; the Observatory was made possible by the generous financial support of the W. M. Keck Foundation.

2 National Optical Astronomy Observatory, 950 North Cherry Avenue, Tucson, AZ 85719-4933; matheson@noao.edu.

${ }^{3}$ European Southern Observatory, Karl-Schwarzschild-Strasse 2, D-85748 Garching, Germany; sblondin@eso.org, bleibund@eso.org, jspyromi@eso.org.

${ }^{4}$ Department of Astronomy, University of California, Berkeley, CA 947203411; rfoley@astro.berkeley.edu, chornock@astro.berkeley.edu, alex@astro .berkeley.edu, sjha@astro.berkeley.edu, weidong@astro.berkeley.edu.

5 National Optical Astronomy Observatory/Cerro Tololo Inter-American Observatory, Casilla 603, La Serena, Chile; csmith@noao.edu, caguilera@ctio .noao.edu, arest@noao.edu,nsuntzeff@noao.edu.
}

the objects so employed are, in fact, SNe Ia. Although the lightcurve shape alone is useful (e.g., Barris \& Tonry 2004), the only way to be sure of the true nature of an object as an SN Ia is through spectroscopy. The calculation of luminosity distances depends on the high-redshift objects being $\mathrm{SNe}$ Ia so that lowredshift calibration methods can be employed. The classification scheme for $\mathrm{SNe}$ is based on their optical spectra, generally near maximum brightness (see Filippenko 1997 for a review of SN types); thus, rest-wavelength optical spectroscopy is

\footnotetext{
${ }^{6}$ Stockholm Observatory, AlbaNova University Center, SE-106 91 Stockholm, Sweden; jesper@astro.su.se.

${ }^{7}$ Harvard-Smithsonian Center for Astrophysics, 60 Garden Street, Cambridge, MA 02138; kirshner@cfa.harvard.edu, pchallis@cfa.harvard.edu, mhicken@cfa .harvard.edu, cstubbs@fas.harvard.edu.

${ }^{8}$ Departamento de Astronomía y Astrofísica, Pontificia Universidad Católica de Chile, Casilla 306, Santiago 22, Chile; aclocchi@astro.puc.cl.

9 Institute for Astronomy, University of Hawaii, 2680 Woodlawn Drive, Honolulu, HI 96822; barris@ifa.hawaii.edu, jt@ifa.hawaii.edu.

${ }^{10}$ Department of Astronomy, University of Washington, Box 351580, Seattle, WA 98195-1580; becker@astro.washington.edu, ricardo@astro.washington.edu, amiceli@astro.washington.edu.

${ }^{11}$ Department of Physics, University of Notre Dame, 225 Nieuwland Science Hall, Notre Dame, IN 46556-5670; pgarnavi@nd.edu, kkrisciu@nd.edu.

12 Department of Physics, Harvard University, 17 Oxford Street, Cambridge, MA 02138.

${ }^{13}$ Department of Physics, University of Washington, Box 351560, Seattle, WA 98195-1560; gm@u.washington.edu.

${ }^{14}$ Department of Astronomy, Ohio State University, $4055 \mathrm{McPherson}$ Laboratory, 140 West 18th Avenue, Columbus, OH 43210; prieto@astronomy ohio-state .edu.

15 Space Telescope Science Institute, 3700 San Martin Drive, Baltimore, MD 21218; ariess@stsci.edu.

${ }^{16}$ Research School of Astronomy and Astrophysics, Australian National University, Mount Stromlo and Siding Spring Observatories, Cotter Road, Weston Creek, ACT 2611, Australia; salvo@mso.anu.edu.au, brian@mso.anu edu.au.
} 
necessary to properly identify SNe Ia at high redshifts. Despite this significance, relatively little attention has been paid to the spectroscopy of high-redshift SNe Ia, with some notable exceptions (Coil et al. 2000). Other publications that include highredshift SN Ia spectra include Schmidt et al. (1998), Riess et al. (1998, 2004b), Perlmutter et al. (1998), Leibundgut \& Sollerman (2001), Tonry et al. (2003), Barris et al. (2004), Blondin et al. (2005), and Lidman et al. (2005).

In addition to providing evidence for the acceleration of the expansion of the universe, it was recognized at an early stage that high-redshift SNe Ia could put constraints on the equation of state of the universe (Garnavich et al. 1998), parameterized as $w=P /\left(\rho c^{2}\right)$, the ratio of the dark energy's pressure to its density. To further explore this, the ESSENCE (Equation of State: SupErNovae trace Cosmic Expansion) project was begun. The ESSENCE project is a 5 yr ground-based SN survey designed to place constraints on the equation-of-state parameter for the universe using $\sim 200 \mathrm{SNe}$ Ia over a redshift range of $0.2 \lesssim z \lesssim 0.8$ (see G. Miknaitis et al. 2005, in preparation, and R. C. Smith et al. 2005, in preparation, for a more extensive discussion of the goals and implementation of the ESSENCE project).

Spectroscopic identification of optical transients is a major component of the ESSENCE project. In addition to confirming some targets as $\mathrm{SNe}$ Ia, the spectroscopy provides redshifts, allowing the derived luminosity distances to be compared with a given cosmological model. So many targets are discovered during the ESSENCE survey that a large amount of telescope time on 6.5-10 m telescopes is required. In the first 2 years of the program, we were fortunate enough to have been awarded over 60 nights at large-aperture telescopes. However, even with this much time, our resources were insufficient to spectroscopically identify all the potentially useful candidates. This remains the most significant limiting factor in achieving the ESSENCE goal of finding, identifying, and following the desired number of $\mathrm{SNe}$ Ia with the appropriate redshift distribution.

Nonetheless, spectroscopic observations of ESSENCE targets in the time available have been successful, with almost $50 \mathrm{SNe}$ Ia clearly identified and several more characterized as likely SNe Ia. Other identifications include core-collapse $\mathrm{SNe}$, active galactic nuclei (AGNs), and galaxies. The galaxy spectra may still include unidentified SN components, and efforts are underway to isolate these through principal component analysis and template subtraction (R. J. Foley et al. 2005, in preparation).

This paper describes the results of the spectroscopic component of the first 2 years of the ESSENCE program. During the campaign, spectroscopically confirmed SNe were announced in IAU Circulars (Smith et al. 2002; Challis 2002, 2003; Covarrubias et al. 2003; Covarrubias 2003; Hicken 2004). "Year One" refers to our 2002 September-December campaign; "Year Two" refers to our 2003 September-December campaign. In $\S 2$ we describe the process of target selection and prioritization. Section 3 describes the technical aspects of the observations. We discuss target identification in $\S 4$. The summary of results in terms of types of objects and success rates is given in $\S 5$. In addition, we present in $\S 5$ all the spectra obtained, including those of the SNe Ia (compared with lowredshift templates), core-collapse $\mathrm{SNe}, \mathrm{AGNs}$, galaxies, stars, and objects that remain unidentified.

\section{TARGET SELECTION}

The ESSENCE survey uses the Blanco $4 \mathrm{~m}$ telescope at the Cerro Tololo Inter-American Observatory with the MOSAIC wide-field CCD camera to detect many kinds of optical transients (R. C. Smith et al. 2005, in preparation). Solar system objects, such as Kuiper Belt objects and asteroids, are identified through their obvious parallax in our multiple observations. Known AGNs and variable stars can also be eliminated from the possible SN Ia list. The remaining transients are all potentially $\mathrm{SNe}$. They are also faint, requiring large-aperture telescopes to obtain spectra of the quality necessary to securely classify the object. Exposure times on 8-10 m telescopes are typically about half an hour, but can be as long as $2 \mathrm{hr}$. Such telescope time is difficult to obtain in quantity, so not all the detected transients can be examined spectroscopically. We apply several criteria to prioritize target selection for spectroscopic observation.

The first step in sorting targets is based on the spectroscopic resources available. The equatorial fields used for the ESSENCE program are accessible from most major astronomical sites, so the main concern with matching targets to spectroscopic telescopes is the aperture size of the telescope. The ESSENCE targets are generally in the range $18 \mathrm{mag} \lesssim m_{R} \lesssim 24 \mathrm{mag}$. When 8-10 m telescopes are unavailable, the fainter targets become lower in priority. The limit for low-dispersion spectroscopy to identify SNe with the $6.5 \mathrm{~m}$ telescopes is $m_{R} \approx 22-23 \mathrm{mag}$, although this varies with weather conditions and seeing. If the full range of telescopes is available, then targets are prioritized by magnitude for observation at a given telescope. The longitudinal distribution of spectroscopic resources can be important if confirmation of a high-priority target is made during a night when multiple spectroscopic resources are available. By the time a target is confirmed, the fields may have set for telescopes in Chile, while they are still accessible from Hawaii. This requires active, real-time collaboration between the group finding SN candidates and those running the spectroscopic observations.

One advantage of the ESSENCE program is that fields are imaged in multiple filters, allowing for discrimination of targets by color. Tonry et al. (2003) present a table of expected SN Ia peak magnitudes as a function of redshift; see also Poznanski et al. (2002), Gal-Yam et al. (2004), Riess et al. (2004a), Strolger et al. (2004), and R. C. Smith et al. (2005, in preparation) for discussions of color selection for SN candidates. Given apparent $R$ - and $I$-band magnitudes, one can calculate the $R-I$ color and compare that with an expected color for those magnitudes. The cadence of the ESSENCE program (returning to the same field every 4 days) will likely catch $\mathrm{SNe}$ at early phases (i.e., before maximum brightness). Early core-collapse SNe are bluer than $\mathrm{SNe}$ Ia, as are AGNs. For example, when selecting for higher redshift targets, objects with $R-I \lesssim 0.2$ mag were considered unlikely to be SNe Ia, while objects with $R-I \gtrsim 0.4$ mag were made high priority for spectroscopic observation. The exact values of $R-I$ used for selection depended on the observed $R$-band magnitude. This method was used more consistently in the last month of Year Two, reducing the fraction of spectroscopic targets that were identified as AGNs from $\sim 10 \%$ over the lifetime of the project to $\sim 5 \%$ during that month.

The cadence of the ESSENCE program is designed to catch SNe early. At the start of an observing campaign or after periods of bad weather, however, we may have missed SNe during their rise to maximum brightness and only caught them while they were declining from maximum. If a target is brightening, then it is a higher priority than one that is not. This prioritization by phase of the SNe became even more important when our Hubble Space Telescope (HST) program to observe some of the ESSENCE SNe Ia was active (see K. Krisciunas et al. 2005, in preparation). The response time of the HST for a new target, 
even if the rough position on the sky was known from our chosen search fields, was still on the order of several days. To ensure that the HST was not generally looking at SNe Ia after maximum brightness, we emphasized targets for spectroscopic identification that appeared to be at an early epoch. In addition, we chose fainter objects, as higher redshift SNe Ia were a prime motivation for HST photometry. The HST observations, while still formally targets of opportunity, were prescheduled for specific ESSENCE search fields, so new targets in those particular fields were given the highest priority.

The position of the SN in the host galaxy also influences the priority for observation. An optical transient located at the core of a galaxy is often an AGN, rather than an SN. The color selection described above is a less biased predictor. In addition, even if the object is an SN, the signal of the SN itself is diluted by the light of the galaxy, making proper identification difficult. Objects that are well separated from the host galaxy are given a higher priority. Being too far from the galaxy can, however, present another problem: the difficulty in obtaining a spectrum of the host in addition to the SN. Without a high signal-to-noise ratio $(\mathrm{S} / \mathrm{N})$ spectrum of the host, there is no precise measure of the redshift. This is especially true if the host galaxy cannot be included in the slit with the target, either to orient the slit at the parallactic angle (Filippenko 1982) or as a result of other observational constraints. In addition, host galaxies can be faint, so the large luminosity contrast with the SN makes detection of the host problematic (the so-called hostless $\mathrm{SNe}$ ), although we did not reject any candidates solely for this reason. The best compromise is to have an object well separated from the host but with the host still in the spectrograph slit. Without narrowline features from the host (either emission or absorption lines), the redshift can be difficult to determine. This lack of a hostgalaxy spectrum became less of a concern, however, as we found that the SN spectrum itself is a relatively accurate, if less precise, measure of the redshift (see discussion below). The light curve alone can be used to estimate distances in a redshiftindependent way (Barris \& Tonry 2004), but only with a wellsampled and accurate light curve.

The target selection process is complex and dynamic. Biases are introduced by some of the steps; for example, SN candidates near the centers of galaxies are less likely to be observed. Since the goal is to optimize the spectroscopic telescope time to identify $\mathrm{SNe}$ Ia in a specific redshift range, we have chosen these selection processes as our best compromise. However, the biases introduced may make the identified sample of SNe Ia problematic for use in statistical studies of the nature of SNe Ia at high redshift.

\section{OBSERVATIONS}

Spectroscopic observations of ESSENCE targets were obtained at a wide variety of telescopes: the Keck I and II 10 m telescopes, the Very Large Telescope (VLT) $8 \mathrm{~m}$, the Gemini North and South $8 \mathrm{~m}$ telescopes, the Magellan Baade and Clay $6.5 \mathrm{~m}$ telescopes, the MMT $6.5 \mathrm{~m}$ telescope, and the Tillinghast $1.5 \mathrm{~m}$ telescope at the F. L. Whipple Observatory (FLWO). The spectrographs used were LRIS (Oke et al. 1995) with Keck I, ESI (Sheinis et al. 2002) with Keck II, FORS1 (Appenzeller et al. 1998) with VLT, GMOS (Hook et al. 2003) with Gemini (North and South), IMACS (Dressler 2004) with Baade, LDSS2 (Mulchaey 2001) with Clay, the Blue Channel (Schmidt et al. 1989) with MMT, and FAST (Fabricant et al. 1998) at FLWO. Nod-and-shuffle techniques (Glazebrook \& Bland-Hawthorn 2001) were used with GMOS (North and South) and IMACS to improve sky subtraction in the red portion of the spectrum.
Standard CCD processing and spectrum extraction were accomplished with IRAF. ${ }^{17}$ Most of the data were extracted using the optimal algorithm of Horne (1986); for the VLT data, an alternative extraction method based on Richardson-Lucy restoration (Blondin et al. 2005) was employed. Low-order polynomial fits to calibration lamp spectra were used to establish the wavelength scale. Small adjustments derived from night-sky lines in the object frames were applied. We employed IRAF and our own IDL routines to flux calibrate the data and, in most cases, to remove telluric lines using the well-exposed continua of the spectrophotometric standards (Wade \& Horne 1988; Matheson et al. 2000).

\section{TARGET IDENTIFICATION}

Once a calibrated spectrum is available, the next step is to properly classify the object. For brighter objects yielding high $\mathrm{S} / \mathrm{N}$ spectra, an SN is often easy to distinguish and classify. Most of the ESSENCE targets are faint enough to be difficult objects even for large-aperture telescopes. The resulting noisy spectra can be confusing. However, even for well-exposed spectra, exact classification can occasionally still be challenging.

For SNe, the classification scheme is based on the optical spectrum, usually near maximum brightness (Filippenko 1997). Type II SNe are distinguished by the presence of hydrogen lines. Type I SNe lack hydrogen and are further subdivided by the presence or absence of other features. The hallmark of SNe Ia is a strong Si II $\lambda 6355$ absorption feature. Near maximum brightness, this absorption is blueshifted by $\sim 10,000 \mathrm{~km} \mathrm{~s}^{-1}$ and appears near $6150 \AA$. In $\mathrm{SNe} \mathrm{Ib}$, this line is not as strong, and the optical helium series dominates the spectrum, especially $\sim 1$ month past maximum brightness. The SNe Ic lack all these identifying lines.

At high redshift, the $\mathrm{Si}$ II $\lambda 6355$ feature is at wavelengths inaccessible to optical spectrographs, so the identification relies on the pattern of features in the rest-frame ultraviolet (UV) and blue optical wavelengths. The Ca II $\mathrm{H}$ and $\mathrm{K} \lambda \lambda 3934,3968$ doublet is a distinctive feature in $\mathrm{SNe} \mathrm{Ia}$, but it is also present in $\mathrm{SNe} \mathrm{Ib} / \mathrm{c}$; therefore, the overall pattern is important for a clear identification as an SN Ia. Other important features to identify SNe Ia include Si II $\lambda 4130, \mathrm{Mg}$ II $\lambda 4481$, Fe II $\lambda 4555, \mathrm{Si}$ III $\lambda 4560, \mathrm{~S}$ II $\lambda 4816$, and Si II $\lambda 5051$ (see, e.g., Jeffery et al. 1992; Kirshner et al. 1993; Mazzali et al. 1993; Coil et al. 2000).

The first stage of classification is done by eye. Drawing on the extensive experience of the spectroscopic observers associated with ESSENCE, we can provide a solid evaluation of the spectrum. Objects such as AGNs and normal galaxies are fairly easy to distinguish. The SNe Ia are also often clear, but some fraction of the data requires more extensive analysis. The first step is simply to make certain that the collective expertise is used, rather than just that of the individual at the telescope. Spectroscopic data are widely disseminated via e-mail and through an internal Web page, allowing rapid examination of any questionable spectrum by the entire collaboration. Broad discussion often leads to a consensus.

In addition to the traditional by-eye approach, we employ automated comparisons. If the object is likely to be an SN Ia, and if the $\mathrm{S} / \mathrm{N}$ is sufficiently high and the rest-wavelength coverage appropriate, we can use a spectral feature aging routine (Riess et al. 1997) that compares specific components of the SN Ia spectrum with a library of SN Ia spectra at known phases. This can pin down the epoch of an SN Ia to within a few days.

\footnotetext{
17 IRAF is distributed by the National Optical Astronomy Observatory, which is operated by AURA under cooperative agreement with the NSF.
} 
However, this program is limited to normal $\mathrm{SNe}$ Ia (i.e., not spectroscopically peculiar objects, which are often overluminous or underluminous). In addition, it does not identify objects that do not match the SNe Ia spectra in the library.

For a more general identification routine, we use an algorithm called SuperNova IDentification (SNID; J. L. Tonry et al. 2005 , in preparation). This program takes the input spectrum and compares it against a library of objects of many types. The templates include SNe Ia of various luminosity classes and a range of ages, core-collapse SNe, and galaxies. The offset in wavelength caused by redshift is a free parameter, so the output includes an estimate of the redshift of the object.

The SNID routine is based on the cross-correlation techniques described by Tonry \& Davis (1979). The input spectrum and the template spectra are binned onto a common logarithmic wavelength scale such that a redshift corresponds to a uniform shift in $\ln \lambda$. The pseudocontinua are removed with a spline fit that effectively discards all color information; thus, the comparison only considers the relative shape of the spectral features. A bandpass filter is applied to the spectra to remove low-frequency residuals left over from the pseudocontinuum subtraction and high-frequency noise components. The input spectrum is crosscorrelated with each of the template spectra in Fourier space, and a fourth-order polynomial is fitted to the highest peak in the resulting cross-correlation function. The center of this fit corresponds to the shift $(\delta)$ in $\ln \lambda$ between the input spectrum and the zeroredshift template, and hence to the redshift of the input spectrum. After this initial identification of the redshift, portions of the template and SN spectra that do not overlap at this redshift are masked out, and the cross-correlation process is repeated. Again, in this refined correlation, a fourth-order polynomial is fitted to the highest peak in the resulting cross-correlation function. The ratio of the peak height $h$ to the antisymmetric part of the correlation function about $\ln \lambda=\delta$ gives the error associated with the output SNID redshift,

$$
r=\frac{h}{2 \sigma_{\text {anti }}},
$$

where $\sigma_{\text {anti }}$ is the rms of the antisymmetric part of the crosscorrelation function; this is normalized such that $h=1$ at zero lag for a perfect spectrum-template match. Thus, $r$ is small $(\sim 1-2)$ for a nonsignificant correlation peak and large $(\geq 5)$ for a good correlation, as $h$ is a significant fraction of 1 and $\sigma_{\text {anti }}$ is small. The above $r$-value is further weighted by the overlap ("lap") in $\ln \lambda$ space between the input spectrum and a given template. For a typical wavelength range of our optical spectra, e.g., $4000 \AA \leq \lambda \leq 9000 \AA$, the maximum value of this overlap is $\operatorname{lap}_{\max }=\ln (9000 / 4000) \approx 0.8$. We thus consider a redshift output by SNID as "good" when

$$
r \times \operatorname{lap} \geq 5
$$

and "poor" otherwise. The error in the redshift found by SNID is taken to be the standard deviation of the template redshifts with the five highest $r \times$ lap values. The phase of the input spectrum is assumed to be equal to that of the best-fit template, i.e., the one with the biggest $r \times$ lap value. Clearly, a more thorough error analysis is needed to investigate the true statistical significance of the $r \times$ lap parameter; this will be done by J. L. Tonry et al. (2005, in preparation). For a subset of the objects $(\leqslant 10 \%)$, the SNID comparison is not optimal. This may be the result of contamination by host-galaxy light, the lack of a matching template in the SNID library, or poor S/ $N$ of the spectrum in ques-

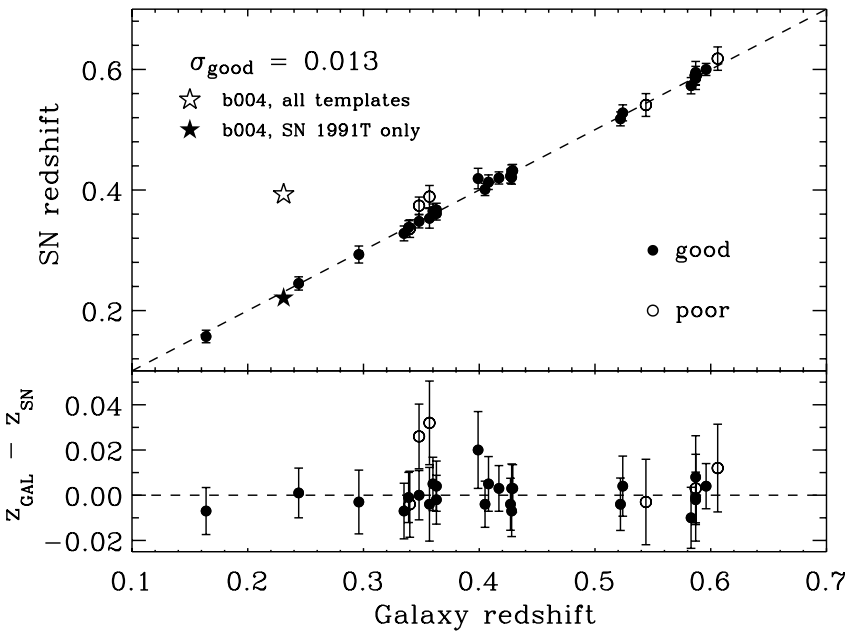

FIG. 1.-Comparison of redshifts as determined by SNID and from narrow emission or absorption lines in the host-galaxy spectrum. Grades for the fits in SNID are described in the text; the good fits (filled circles) are shown, as well as the poor fits (open circles). The dispersion around one-to-one correspondence of the redshifts for the good data is excellent, with $\sigma=0.013$. Errors include 0.01 in $z$ added in quadrature to account for potential effects on the cross-correlation related to unusual velocity features in the spectra. There is one outlier (b004) for which the redshift determination using SNID is highly degenerate, as it is likely to be a peculiar SN Ia (see text); for the sake of clarity, we do not show b004 in the residual plot. Note that the mean residual is $\sim 10^{-4} \ll \sigma_{\text {good }}$, which shows that there are no systematic effects associated with the use of SNID in determining the SN redshift.

tion. All SNID comparisons are checked by eye for a qualitative judgment of the goodness of fit.

The redshift of the object can also be directly determined from the spectrum itself if narrow emission or absorption lines associated with the host galaxy are present. Occasionally, observations are set up to include the host galaxy in the spectrograph slit specifically for the purpose of obtaining a redshift. If there is a strong enough signal of a galaxy spectrum but no clearly identifiable narrow emission or absorption lines, crosscorrelation with an absorption template can be used. For the spectra that have narrow emission or absorption lines (or were cross-correlated with a template), we report the redshift to three significant digits. If the redshift determination is based solely on a comparison of the $\mathrm{SN}$ spectrum to a low-redshift analog, the redshift is less certain, and we only report the value to two significant digits. The uncertainty of 0.01 in redshift is fully vindicated by the results in Figure 1.

For the objects with a more precise redshift derived from the host galaxy, we can compare the galactic redshift with the value of the redshift estimated by SNID. Figure 1 shows that the SNID redshifts agree well with the galaxy redshifts. Tests with template spectra that have unusual line shapes or high-velocity features indicate that the derived redshift is not affected significantly, but we do include possible effects of these spectroscopic features in the errors of the derived redshifts. Thus, for objects without precise redshifts from host-galaxy spectra, the SNID redshifts can be used as reliable substitutes. In cases in which SNID preferred an SN redshift significantly different from the host-galaxy redshift, we classified the SN by forcing its redshift to equal that of the host galaxy. The only object for which this was necessary is b004 (see below).

\section{RESULTS}

The results of our spectroscopic observations during the first 2 years of the ESSENCE program are summarized in Table 1. 
TABLE 1

ESSENCE Spectroscopy Results: The First 2 Years

\begin{tabular}{|c|c|c|c|}
\hline Type $^{\mathrm{a}}$ & Year 1 & Year 2 & Total \\
\hline 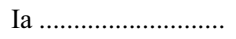 & 15 & 31 & 46 \\
\hline Ia?............ & 0 & 6 & 6 \\
\hline II $\ldots \ldots \ldots \ldots \ldots \ldots \ldots \ldots$ & 2 & 2 & 4 \\
\hline 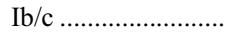 & 0 & 1 & 1 \\
\hline AGN ....................... & 4 & 8 & 12 \\
\hline Gal........................... & 7 & 12 & 19 \\
\hline Star ........................... & 2 & 2 & 4 \\
\hline N.S. .................... & 5 & 5 & 10 \\
\hline Unk........................ & 2 & 14 & 16 \\
\hline N.A.................... & 13 & 43 & 54 \\
\hline Total ................... & 50 & 124 & 174 \\
\hline
\end{tabular}

a Our best guess as to classification of the object. Ia? indicates a lack of certainty in the identification as an SN Ia. N.S. indicates that the telescope was pointed at the object, but no spectrum was obtained or the exposure contained essentially no signal. Unk. represents objects for which we have spectra but are uncertain as to their classification. N.A. indicates that a transient was found in the ESSENCE search, but no attempt to take a spectrum was made, either because it was a poor target or there were not enough spectroscopic resources available.

There are $46 \mathrm{SNe}$ Ia (and six additional likely SNe Ia), along with five core-collapse SNe. Note also that there were 54 transients in the first 2 years that were not observed spectroscopically, so the yield of SNe Ia is 52 out of 120 objects. Through the target selection methods described in $\S 2$, we were able to prioritize the more likely candidates, but many of these were not observed simply because of the lack of sufficient spectroscopic resources. This became more of an issue toward the end of Year Two, when good weather and increasingly efficient detection algorithms increased the number of transients discovered.

The goal of the ESSENCE project is to find $\sim 200$ SNe Ia over the redshift range $0.2 \lesssim z \lesssim 0.8$. In Figure 2 we show the actual distribution in redshift of the SNe Ia from the ESSENCE project

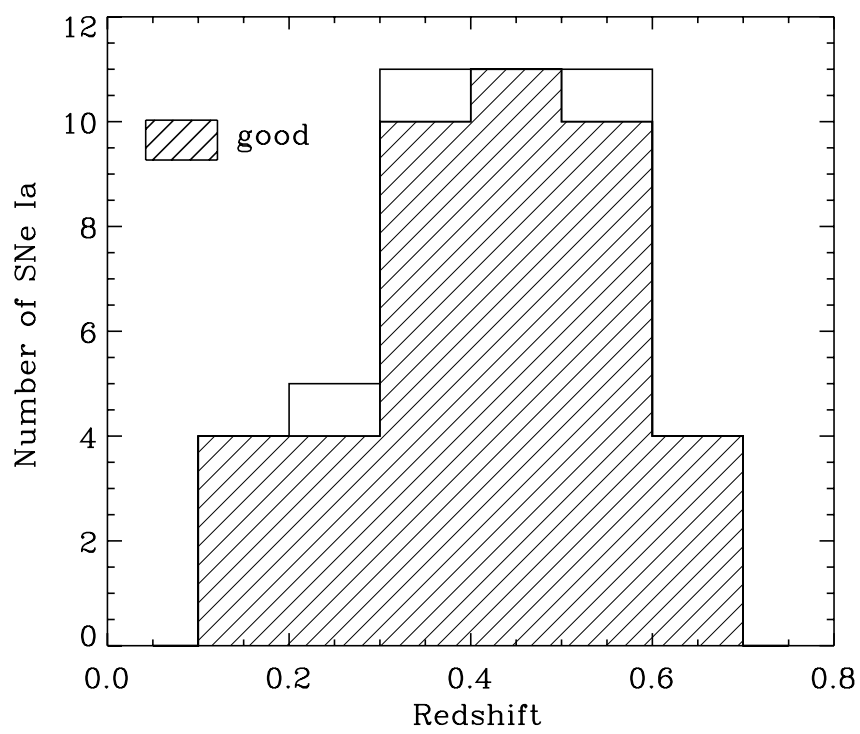

FIG. 2.-Redshift distribution of definite, spectroscopically identified $\mathrm{SNe}$ Ia from the first 2 years of the ESSENCE project. The SNe for which the SNID fit is good (see text) are indicated by the hatched area, while those that are poor fits are indicated by the blank areas.

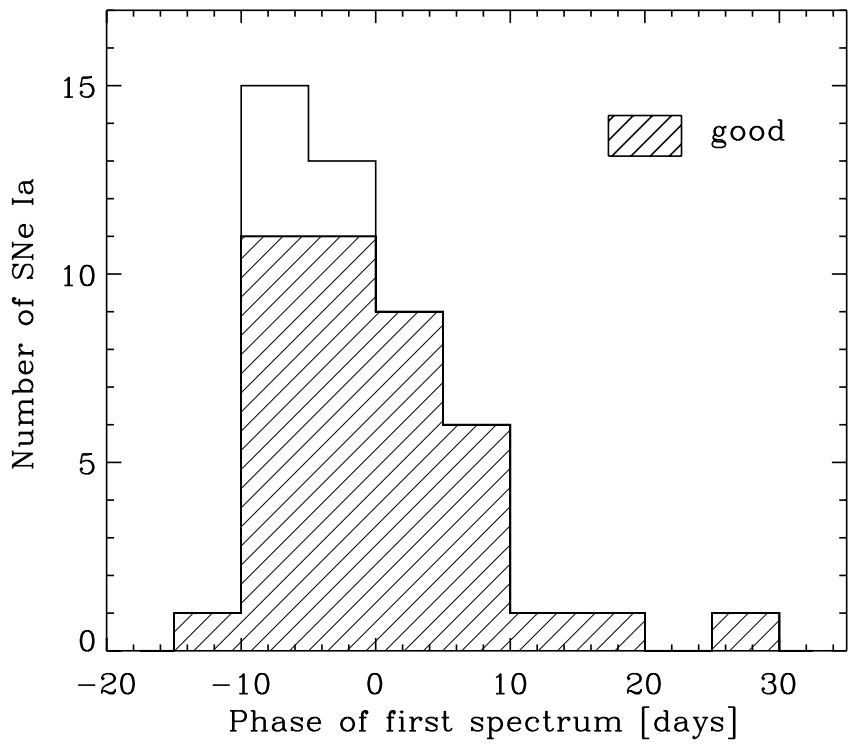

FIG. 3.-Age distribution (relative to maximum brightness) of definite, spectroscopically identified SNe Ia from the first 2 years of the ESSENCE project. Ages are determined from spectroscopic features alone. The SNe for which the SNID fit is good (see text) are indicated by the hatched areas, while those that are poor fits are indicated by the blank area. For objects with multiple epochs of spectroscopy, this figure only reflects the first spectrum.

that are spectroscopically confirmed. There are $\mathrm{SNe}$ over most of the targeted redshift range, although there are relatively fewer at the high end $(z \gtrsim 0.6)$. A significant fraction of the signal of $w$ is accessible at $z \approx 0.5$ (G. Miknaitis et al. 2005, in preparation), but a goal for the next 3 years of the program is to ensure that the $\mathrm{SNe}$ Ia observed spectroscopically are distributed optimally over our targeted redshift range. This highlights the importance of the 8-10 m telescopes such as Gemini, the VLT, and Keck that are critical to spectroscopy of the faint objects at the high-redshift end of our range.

Both SNID and the spectral feature aging method described in $\S 4$ give an indication of the age of the SN Ia. Light curves provide a more precise measure of the age of the $\mathrm{SN}$ at the time of the spectroscopy, but an estimate of the epoch of the spectrum to within a few days is possible from the spectral features alone. Figure 3 shows the distribution in age (relative to maximum brightness) at the time of spectroscopy (not discovery, as spectra are often taken up to several days after discovery). In the 15 cases $^{18}$ for which we have spectra of the same SN Ia at multiple epochs, the relative ages are consistent with the times of the spectroscopic exposures (also considering the effects of cosmological time dilation and probable errors of the fits of $\sim \pm 3$ days). There is one exception to this consistency (b027), but at a later epoch when the uncertainty in the estimated spectroscopic age increases as a result of slower spectral evolution.

Table 2 gives all ESSENCE targets that were selected for spectroscopic identification. The results for these first 2 years include $52 \mathrm{SNe}$ Ia or likely SNe Ia (Figs. 4-10), four SNe II (Fig. 11), one SN Ib/c (Fig. 11), 12 AGNs (Figs. 12 and 13), four possibly variable stellar objects (Fig. 14), 19 galaxies (Figs. 15 and 16), and 16 objects of unknown classification (Figs. 17 and 18). There were 10 objects for which we pointed the telescope at the target and did not get a spectrum, either

\footnotetext{
18 These are b008, b010, b013, b020, b022, b023, b027, c003, c012, c015, d086, d093, e029, e108, and f076.
} 
TABLE 2

ESSENCE Spectroscopic Targets: The First 2 Years

\begin{tabular}{|c|c|c|c|c|c|c|c|c|c|c|c|}
\hline ESSENCE ID ${ }^{\mathrm{a}}$ & IAUC ID $^{\mathrm{b}}$ & UT Date $^{\mathrm{c}}$ & Telescope & Type $^{\mathrm{d}}$ & $\begin{array}{c}z \\
(\text { Gal. })^{\mathrm{e}}\end{array}$ & $\begin{array}{c}z \\
(\mathrm{SNID})^{\mathrm{f}}\end{array}$ & $\begin{array}{c}\text { Epoch } \\
(\mathrm{SNID})^{\mathrm{g}}\end{array}$ & Template $^{\mathrm{h}}$ & Grade $^{\mathrm{i}}$ & $\begin{array}{l}\text { Discovery }{ }^{\mathrm{j}} \\
\quad(\mathrm{mag})\end{array}$ & $\begin{array}{l}\text { Exp. } \\
\text { (s) }\end{array}$ \\
\hline a002.wxc1_04......... & $\ldots$ & 2002 Dec 6.03 & VLT & Gal & 0.316 & $\ldots$ & $\ldots$ & $\ldots$ & $\ldots$ & $\ldots$ & 900 \\
\hline b001.wxc1_14 ........ & $\ldots$ & 2002 Nov 6.27 & KII/ESI & Unk & $\ldots$ & $\ldots$ & $\ldots$ & $\ldots$ & $\ldots$ & 23.6 & 1600 \\
\hline b001.wxc1_14 ........ & $\ldots$ & 2002 Nov 11.32 & KI/LRIS & Unk & $\ldots$ & $\ldots$ & $\ldots$ & $\ldots$ & $\ldots$ & 23.6 & 1800 \\
\hline b001.wxc1_14 ........ & $\ldots$ & 2002 Dec 5.26 & GMOS & Unk & $\ldots$ & $\ldots$ & $\ldots$ & $\ldots$ & $\ldots$ & 23.6 & $2 \times 1800$ \\
\hline b002.wxh1_01 ........ & $\ldots$ & 2002 Nov 1.44 & KII/ESI & Star & $\ldots$ & $\ldots$ & $\ldots$ & $\ldots$ & $\ldots$ & $\ldots$ & 900 \\
\hline b003.wxh1_14 ........ & $2002 \mathrm{iu}$ & 2002 Nov 1.43 & $\mathrm{KII} / \mathrm{ESI}$ & Ia & $\cdots$ & 0.11 & 2 & $94 \mathrm{~S}$ & Good & 18.9 & 600 \\
\hline b004.wxt2_06 ......... & $2002 \mathrm{iv}$ & 2002 Nov 2.45 & KII/ESI & Ia & 0.231 & 0.39 & -4 & $95 \mathrm{ac}$ & Good & 20.9 & 1200 \\
\hline b005.wxd1_11 ........ & $2002 \mathrm{iw}$ & 2002 Nov 3.16 & MMT & Gal & 0.205 & $\ldots$ & $\ldots$ & $\ldots$ & $\ldots$ & 21.8 & $3 \times 1800$ \\
\hline b005.wxd1_11 ........ & $2002 \mathrm{iw}$ & 2002 Nov 6.32 & KII/ESI & Gal & 0.205 & $\ldots$ & $\ldots$ & $\ldots$ & $\ldots$ & 21.8 & 1800 \\
\hline b006.wxb1_16 ........ & $2002 \mathrm{ix}$ & 2002 Nov 3.10 & MMT & N.S. & $\ldots$ & $\ldots$ & $\ldots$ & $\ldots$ & $\ldots$ & 22.2 & $\ldots$ \\
\hline b006.wxb1_16 ........ & $2002 \mathrm{ix}$ & 2002 Nov 6.24 & KII/ESI & II? & $\ldots$ & $\ldots$ & $\ldots$ & $\ldots$ & $\ldots$ & 22.2 & 1800 \\
\hline b008.wxc1_05 ........ & $2002 j q$ & 2002 Nov 6.29 & KII/ESI & Ia & $\ldots$ & 0.49 & -8 & $90 \mathrm{~N}$ & Good & 21.9 & 1800 \\
\hline b008.wxc1_05 ........ & $2002 \mathrm{jq}$ & 2002 Dec 4.27 & GMOS & Ia & $\ldots$ & 0.49 & +13 & 89B & Good & 21.9 & $4 \times 1800$ \\
\hline b010.wxv2_07 ........ & 2002iy & 2002 Nov 6.39 & KII/ESI & Ia & $\ldots$ & 0.59 & -1 & $92 \mathrm{~A}$ & Poor & 21.3 & 1800 \\
\hline b010.wxv2_07 ........ & 2002iy & 2002 Nov 11.45 & KI/LRIS & Ia & $\ldots$ & 0.59 & +2 & $94 \mathrm{ae}$ & Good & 21.3 & 1800 \\
\hline b010.wxv2_07 ........ & 2002iy & 2002 Dec 6.35 & GMOS & Ia & $\ldots$ & 0.59 & +13 & $89 \mathrm{~B}$ & Good & 21.3 & $5 \times 1800$ \\
\hline b010.wxv2_07 ........ & 2002iy & 2002 Dec 7.22 & VLT & Ia & 0.587 & 0.59 & +17 & 95al & Good & 21.3 & $2 \times 1800$ \\
\hline b013.wxv2_10 ........ & $2002 \mathrm{iz}$ & 2002 Nov 6.45 & KII/ESI & Ia & 0.427 & 0.42 & -6 & $90 \mathrm{~N}$ & Good & 22.1 & 1800 \\
\hline b013.wxv2_10 ........ & $2002 \mathrm{iz}$ & 2002 Dec 6.07 & VLT & Ia & 0.428 & 0.43 & +14 & $89 B$ & Good & 22.1 & 1800 \\
\hline b014.wxv2_15 ........ & $\ldots$ & 2002 Nov 6.41 & KII/ESI & Gal & 0.268 & $\ldots$ & $\ldots$ & $\ldots$ & $\ldots$ & 22.8 & 1800 \\
\hline b015.wcx1_09 ........ & $\ldots$ & 2002 Nov 6.34 & KII/ESI & Gal & 0.207 & $\ldots$ & $\ldots$ & $\ldots$ & $\ldots$ & $\ldots$ & 1800 \\
\hline b016.wxb1_15 ........ & $2002 \mathrm{ja}$ & 2002 Nov 9.33 & KII/ESI & $\mathrm{Ia}$ & $\ldots$ & 0.33 & +2 & $94 \mathrm{ae}$ & Good & 22.2 & 1200 \\
\hline b017.wxb1_06 ........ & $2002 \mathrm{jb}$ & 2002 Nov 9.31 & KII/ESI & Ia & $\ldots$ & 0.25 & +2 & $94 \mathrm{ae}$ & Good & 21.4 & 1200 \\
\hline b019.wxd1_04 ........ & $\ldots$ & 2002 Nov 9.35 & KII/ESI & Gal & 0.213 & $\ldots$ & $\ldots$ & $\ldots$ & $\ldots$ & 22.2 & 1200 \\
\hline b020.wye2_01 ........ & $2002 \mathrm{jr}$ & 2002 Nov 9.42 & KII/ESI & Ia & $\ldots$ & 0.43 & -9 & $91 \mathrm{M}$ & Poor & 22.7 & 1800 \\
\hline b020.wye2_01 ........ & $2002 \mathrm{jr}$ & 2002 Dec 9.39 & GMOS & Ia & $\ldots$ & 0.43 & +7 & $72 \mathrm{E}$ & Good & 22.7 & $2 \times 1800$ \\
\hline b022.wyc3_03 ........ & $2002 \mathrm{jc}$ & 2002 Nov 9.45 & KII/ESI & Ia & $\ldots$ & 0.52 & -7 & $90 \mathrm{~N}$ & Poor & 23.1 & 1800 \\
\hline b022.wyc3_03 ........ & $2002 \mathrm{jc}$ & 2002 Dec 5.39 & GMOS & $\mathrm{Ia}$ & $\cdots$ & 0.52 & +4 & $96 Z$ & Good & 23.1 & $4 \times 1800$ \\
\hline b023.wxu2_09 ........ & $2002 \mathrm{js}$ & 2002 Nov 11.57 & KI/LRIS & Ia & $\ldots$ & 0.54 & -7 & 89B & Poor & 22.9 & 2100 \\
\hline b023.wxu2_09 ........ & $2002 \mathrm{js}$ & 2002 Dec 3.41 & GMOS & Ia & $\ldots$ & 0.54 & +6 & $92 \mathrm{~A}$ & Good & 22.9 & $4 \times 1800$ \\
\hline b024.wxc1_16 ........ & $\ldots$ & 2002 Nov 11.27 & KI/LRIS & VStar & $\ldots$ & $\ldots$ & $\ldots$ & $\ldots$ & $\ldots$ & 21.5 & 1800 \\
\hline b025.wxa1_05 ........ & $\ldots$ & 2002 Nov 11.29 & KI/LRIS & N.S. & $\ldots$ & $\ldots$ & $\ldots$ & $\ldots$ & $\ldots$ & 22.2 & 1800 \\
\hline b026.wxk1_05 ........ & $\ldots$ & 2002 Nov 11 & KI/LRIS & N.S. & $\ldots$ & $\ldots$ & $\ldots$ & $\ldots$ & $\ldots$ & 22.2 & $\ldots$ \\
\hline b027.wxm1_16 ....... & $2002 \mathrm{jd}$ & 2002 Nov 11.45 & KI/LRIS & Ia & $\ldots$ & 0.32 & +0 & $81 \mathrm{~B}$ & Good & 22.0 & $3600,1800^{\mathrm{k}}$ \\
\hline b027.wxm1_16 ....... & $2002 \mathrm{jd}$ & 2002 Dec 6.07 & VLT & Ia & $\ldots$ & 0.32 & +13 & 89B & Good & 22.0 & 1800 \\
\hline b027.wxm1_16 ...... & $2002 \mathrm{jd}$ & 2002 Dec 9.27 & GMOS & Ia & $\ldots$ & 0.32 & +12 & $92 \mathrm{G}$ & Poor & 22.0 & $4 \times 1800$ \\
\hline c002.wxp1_14 ........ & $\ldots$ & 2002 Dec 1 & Clay & N.S. & $\cdots$ & $\ldots$ & $\ldots$ & $\ldots$ & $\ldots$ & 22.4 & $\ldots$ \\
\hline c002.wxp1_14 ........ & $\ldots$ & 2002 Dec 3 & GMOS & N.S. & $\ldots$ & $\ldots$ & $\ldots$ & $\ldots$ & $\ldots$ & 22.4 & $\ldots$ \\
\hline c003.wxh1_15 ........ & $2002 \mathrm{jt}$ & 2002 Dec 2.13 & Clay & Ia & $\ldots$ & 0.56 & -7 & $89 B$ & Good & 22.6 & $2 \times 1800$ \\
\hline c003.wxh1_15 ........ & $2002 \mathrm{jt}$ & 2002 Dec 7.29 & GMOS & Ia & $\ldots$ & 0.56 & +0 & $94 \mathrm{~S}$ & Poor & 22.6 & $3 \times 1800$ \\
\hline c005.wxb1_10 ........ & $\ldots$ & 2002 Dec 6.05 & VLT & AGN & 0.249 & $\ldots$ & $\ldots$ & $\ldots$ & $\ldots$ & $\ldots$ & 900 \\
\hline c012.wxu2_ $16^{1} \ldots \ldots .$. & $2002 \mathrm{ju}$ & 2002 Dec 3.15 & Clay & Ia & 0.348 & 0.35 & -8 & $90 \mathrm{~N}$ & Good & 21.6 & $2 \times 1800$ \\
\hline c012.wxu2_16 $16^{1} \ldots \ldots . .$. & $2002 \mathrm{ju}$ & 2002 Dec 4.43 & GMOS & $\mathrm{Ia}$ & 0.348 & 0.35 & -8 & $90 \mathrm{~N}$ & Good & 21.6 & $3 \times 1200$ \\
\hline c012.wxu2_16 ........ & $2002 \mathrm{ju}$ & 2003 Jan 5.33 & KII/ESI & Ia & 0.348 & 0.35 & +14 & 89B & Poor & 21.6 & 1800 \\
\hline c013.wxm1_13 „...... & $\ldots$ & 2002 Dec 5 & GMOS & N.S. & $\ldots$ & $\ldots$ & $\ldots$ & $\ldots$ & $\ldots$ & $\ldots$ & $\ldots$ \\
\hline c014.wyb3_03 ........ & $2002 \mathrm{jv}$ & 2002 Dec 7.12 & VLT & Gal & 0.221 & $\ldots$ & $\ldots$ & $\ldots$ & $\ldots$ & 22.6 & $2 \times 1800$ \\
\hline c014.wyb3_03 ........ & $2002 \mathrm{jv}$ & 2003 Jan 4.28 & GMOS & Gal & 0.221 & $\ldots$ & $\ldots$ & $\ldots$ & $\ldots$ & 22.6 & $4 \times 1800$ \\
\hline c015.wxv2_02 ........ & $2002 \mathrm{jw}$ & 2002 Dec 7.18 & VLT & Ia & 0.357 & 0.35 & +0 & $81 \mathrm{~B}$ & Good & 22.8 & $2 \times 1800$ \\
\hline c015.wxv2_02 ........ & $2002 \mathrm{jw}$ & 2003 Jan 5.37 & KII/ESI & Ia & 0.356 & 0.38 & +12 & $89 B$ & Poor & 22.8 & 2400 \\
\hline c016.wxm1_04 ....... & $\ldots$ & 2002 Dec 7.07 & VLT & AGN & 0.845 & $\ldots$ & $\ldots$ & $\ldots$ & $\ldots$ & 23.5 & $2 \times 1800$ \\
\hline c020.wxt2_15 ......... & $\ldots$ & 2003 Jan 5.30 & KII/ESI & Unk & 0.650 & $\ldots$ & $\ldots$ & $\ldots$ & $\ldots$ & 23.3 & $2 \times 900$ \\
\hline c020.wxt2_-15 ......... & $\ldots$ & 2003 Jan 10.11 & VLT & Unk & $\ldots$ & $\ldots$ & $\cdots$ & $\cdots$ & $\ldots$ & 23.3 & $2 \times 1800$ \\
\hline c022.wxu2_15 ........ & $\ldots$ & 2003 Jan 4.09 & Clay & II? & 0.213 & $\ldots$ & $\ldots$ & $\ldots$ & $\ldots$ & $\ldots$ & $2 \times 1200$ \\
\hline c023.wxm1_15 ....... & $\cdots$ & 2003 Jan 3.07 & Clay & Ia & 0.399 & 0.42 & -8 & $90 \mathrm{~N}$ & Poor & $\cdots$ & $2 \times 1200$ \\
\hline c024.wxv2_05 ........ & $\ldots$ & 2003 Jan 3.12 & Clay & Gal & 0.317 & $\ldots$ & $\ldots$ & $\ldots$ & $\ldots$ & $\ldots$ & 1800 \\
\hline c025.wxb1_14 ........ & $\ldots$ & 2003 Jan 4.05 & Clay & AGN & 0.362 & $\ldots$ & $\ldots$ & $\ldots$ & $\ldots$ & $\ldots$ & 600 \\
\hline c028.wxu2_16 ........ & $\ldots$ & 2003 Jan 4.13 & Clay & AGN & 2.02 & $\ldots$ & $\ldots$ & $\ldots$ & $\ldots$ & $\ldots$ & 1800 \\
\hline d009.waa6_16 ${ }^{\mathrm{m}} \ldots \ldots$ & $\cdots$ & 2003 Oct 29.11 & VLT & Gal & 0.352 & $\cdots$ & $\cdots$ & $\cdots$ & $\cdots$ & $\cdots$ & 1800 \\
\hline d106.waa6_16 ${ }^{\mathrm{m}} \ldots \ldots$. & $\ldots$ & 2003 Oct 31.01 & VLT & Gal & 0.353 & $\ldots$ & $\ldots$ & $\ldots$ & $\ldots$ & $\ldots$ & 1800 \\
\hline d010.waa6_16......... & 2003jp & 2003 Oct 30.03 & VLT & $\mathrm{Ib} / \mathrm{c}$ & $\ldots$ & 0.08 & +35 & $87 \mathrm{M}^{\mathrm{n}}$ & Poor & 21.6 & $2 \times 1800$ \\
\hline d029.waa6_13......... & $\ldots$ & 2003 Oct 29.03 & VLT & AGN & 2.575 & $\ldots$ & $\ldots$ & $\ldots$ & $\ldots$ & 21.6 & $2 \times 1800$ \\
\hline d033.waa6_10......... & 2003jo & 2003 Oct 29.09 & VLT & Ia & 0.524 & 0.53 & -1 & $89 B$ & Good & 20.9 & $2 \times 1800$ \\
\hline d033.waa6_10......... & 2003jo & 2003 Nov 23.05 & VLT & N.S. & 0.524 & $\ldots$ & $\cdots$ & $\ldots$ & $\ldots$ & 20.9 & $2 \times 1800$ \\
\hline d034.waa7_10......... & $\ldots$ & 2003 Oct 28.29 & GMOS & AGN & 2.28 & $\ldots$ & $\ldots$ & $\ldots$ & $\ldots$ & 21.4 & $2 \times 1200$ \\
\hline
\end{tabular}


TABLE 2-Continued

\begin{tabular}{|c|c|c|c|c|c|c|c|c|c|c|c|}
\hline ESSENCE ID $^{\mathrm{a}}$ & IAUC ID ${ }^{\mathrm{b}}$ & UT Date $^{\mathrm{c}}$ & Telescope & Type $^{d}$ & $\begin{array}{c}z \\
(\mathrm{Gal} .)^{\mathrm{e}}\end{array}$ & $\begin{array}{c}z \\
(\mathrm{SNID})^{\mathrm{f}}\end{array}$ & $\begin{array}{c}\text { Epoch } \\
\text { (SNID) }^{\mathrm{g}}\end{array}$ & Template $^{\mathrm{h}}$ & Grade $^{\mathrm{i}}$ & $\begin{array}{l}\text { Discovery }{ }^{\mathrm{j}} \\
\quad(\mathrm{mag})\end{array}$ & $\begin{array}{l}\text { Exp. } \\
\text { (s) }\end{array}$ \\
\hline d051.wcc8_2 2.......... & & 2003 Oct 30.22 & VLT & Gal & 0.382 & $\ldots$ & $\ldots$ & $\ldots$ & $\ldots$ & $\ldots$ & 1800 \\
\hline d057.wbb6_3 ........... & $2003 \mathrm{jk}$ & 2003 Oct 30.15 & VLT & Unk & & $\ldots$ & $\ldots$ & $\ldots$ & $\ldots$ & 20.9 & $2 \times 1800$ \\
\hline d058.wbb6_3 ........... & $2003 \mathrm{jj}$ & 2003 Oct 31.07 & VLT & Ia & 0.583 & 0.58 & -1 & $92 \mathrm{~A}$ & Good & 23.1 & $2 \times 1800$ \\
\hline d059.wcc5_3........... & $\ldots$ & 2003 Oct 29.17 & VLT & Gal & 0.207 & $\ldots$ & $\ldots$ & $\ldots$ & $\ldots$ & 19.2 & $2 \times 1800$ \\
\hline d060.wcc7_3 ............. & $\ldots$ & 2003 Oct 30.35 & VLT & M star & $\ldots$ & $\ldots$ & $\ldots$ & $\ldots$ & $\ldots$ & $\ldots$ & 1800 \\
\hline d062.wcc9_3........... & $\ldots$ & 2003 Oct 29.26 & VLT & $\mathrm{AGN}$ & 2.42 & $\ldots$ & $\ldots$ & $\ldots$ & $\ldots$ & 20.3 & 1800 \\
\hline d083.wdd9_12 ......... & 2003jn & 2003 Oct 29.29 & VLT & Ia & $\ldots$ & 0.33 & -1 & $91 \mathrm{~T}$ & Good & 20.8 & 1800 \\
\hline d084.wdd9_11 ......... & $2003 \mathrm{jm}$ & 2003 Oct 30.19 & VLT & Ia & 0.522 & 0.52 & +8 & $72 \mathrm{E}$ & Good & 22.9 & 1800 \\
\hline d085.waa5_16.......... & $2003 \mathrm{jv}$ & 2003 Oct 28.37 & GMOS & Ia & 0.405 & 0.41 & +3 & $94 \mathrm{ae}$ & Good & 22.2 & $3 \times 1200$ \\
\hline d086.waa5_3 ............. & $2003 \mathrm{ju}$ & 2003 Oct 27.06 & GMOS & Ia & $\ldots$ & 0.20 & -7 & $89 \mathrm{~B}$ & Poor & 21.6 & $3 \times 600$ \\
\hline d086.waa5_3 ............ & $2003 \mathrm{ju}$ & 2003 Nov 27.10 & Baade & Ia & $\ldots$ & 0.20 & +13 & $89 \mathrm{~B}$ & Good & 21.6 & $3 \times 1800$ \\
\hline d087.wbb5_4 ........... & $2003 \mathrm{jr}$ & 2003 Nov 1.18 & GMOS & Ia & 0.340 & 0.34 & +6 & $95 \mathrm{E}$ & Good & 21.9 & $3 \times 600$ \\
\hline d089.wdd6_8 ........... & 2003jl & 2003 Oct 31.34 & VLT & Ia & 0.429 & 0.43 & +6 & $95 \mathrm{E}$ & Good & 22.4 & 1800 \\
\hline d091.wcc1_2.. & $\ldots$ & 2003 Oct 29.22 & VLT & Unk & $\ldots$ & $\ldots$ & $\ldots$ & $\ldots$ & $\ldots$ & $\ldots$ & $2 \times 1800$ \\
\hline d093.wdd5_3 ${ }^{\circ} \ldots \ldots \ldots . .$. & $2003 \mathrm{js}$ & 2003 Oct 29.96 & VLT & Ia & 0.363 & 0.36 & -6 & $90 \mathrm{~N}$ & Good & 22.0 & $923+600$ \\
\hline e142.wdd5_3ㅇ…...... & $2003 \mathrm{js}$ & 2003 Nov 23.21 & VLT & Ia & 0.363 & 0.36 & +12 & $95 \mathrm{D}$ & Good & 22.0 & $3 \times 1200$ \\
\hline d097.wdd5_10 ......... & $2003 \mathrm{jt}$ & 2003 Oct 29.32 & VLT & Ia & $\ldots$ & 0.45 & -5 & 900 & Good & 22.0 & 1800 \\
\hline d099.wcc2_16......... & $2003 \mathrm{ji}$ & 2003 Nov 1.23 & GMOS & Ia & $\ldots$ & 0.21 & +17 & $95 \mathrm{bd}$ & Good & 20.9 & $3 \times 600$ \\
\hline d100.waa7_16.......... & $2003 j q$ & 2003 Oct 24.21 & FLWO & Ia & $\ldots$ & 0.16 & +26 & $95 \mathrm{al}$ & Good & 19.8 & $2 \times 1800$ \\
\hline d115.wbb6_11 ......... & & 2003 Oct 28.43 & GMOS & Unk & $\ldots$ & $\ldots$ & $\ldots$ & $\ldots$ & & 20.2 & 1200 \\
\hline d117.wdd8_16 ......... & $2003 \mathrm{jw}$ & 2003 Oct 30.32 & VLT & Ia & 0.296 & 0.29 & -1 & $95 \mathrm{E}$ & Good & 22.6 & 1800 \\
\hline d123.wcc9_16.......... & $\ldots$ & 2003 Oct 30.27 & VLT & Gal & 0.500 & $\ldots$ & $\ldots$ & $\ldots$ & $\ldots$ & $\ldots$ & $\ldots$ \\
\hline d124.wcc9_15.......... & $\ldots$ & 2003 Oct 31.26 & VLT & AGN & 0.609 & $\ldots$ & $\ldots$ & $\ldots$ & $\ldots$ & 20.5 & 1800 \\
\hline d149.wcc4_11 .......... & 2003jy & 2003 Oct 31.10 & VLT & Ia & 0.339 & 0.34 & -5 & 900 & Good & 22.7 & 1800 \\
\hline d150.wcc1_12......... & $\ldots$ & 2003 Oct 31.31 & VLT & Gal & 0.190 & $\ldots$ & $\ldots$ & $\ldots$ & $\ldots$ & $\ldots$ & $\ldots$ \\
\hline d156.wcc2_4........... & $2003 j x$ & 2003 Oct 31.15 & VLT & Unk & $\ldots$ & $\ldots$ & $\ldots$ & $\ldots$ & $\ldots$ & $\ldots$ & $2 \times 1800$ \\
\hline e018.wbb7_2 …........ & $\ldots$ & 2003 Nov 19.05 & Clay & AGN & 0.181 & $\ldots$ & $\ldots$ & $\ldots$ & $\ldots$ & 18.6 & 600 \\
\hline e020.waa6_9............. & $2003 \mathrm{kk}$ & 2003 Nov 19.11 & Clay & Ia & 0.164 & 0.16 & -5 & 900 & Good & 20.3 & $3 \times 300$ \\
\hline e022.wbb7_12 .......... & $2003 \mathrm{kj}$ & 2003 Nov 22.03 & VLT & II & 0.074 & $\ldots$ & $\ldots$ & $\ldots$ & $\ldots$ & 22.3 & $1800+900$ \\
\hline e025.wdd3_15 ......... & $\ldots$ & 2003 Nov 19.21 & Clay & Gal & 0.180 & $\ldots$ & $\ldots$ & $\ldots$ & $\ldots$ & $\ldots$ & $3 \times 1200$ \\
\hline e027.wcc7_16 .......... & $\ldots$ & 2003 Nov 21.16 & VLT & Unk & $\ldots$ & $\ldots$ & $\ldots$ & $\ldots$ & $\ldots$ & 22.5 & $3 \times 1200$ \\
\hline e029.wbb3_15 $5^{\mathrm{p}} \ldots \ldots .$. & $2003 \mathrm{kl}$ & 2003 Nov 19.14 & Clay & Ia & 0.335 & 0.33 & -5 & $90 \mathrm{O}$ & Good & 21.0 & $3 \times 600$ \\
\hline e121.wbb3_15 $5^{\mathrm{p}} \ldots \ldots .$. & $2003 \mathrm{kl}$ & 2003 Nov 22.11 & Clay & Ia & 0.335 & 0.33 & -1 & $81 \mathrm{~B}$ & Good & 21.0 & $3 \times 1200$ \\
\hline e103.wbb9_2 …........ & $\ldots$ & 2003 Nov 21.05 & VLT & Unk & 0.871 & $\ldots$ & $\ldots$ & $\ldots$ & $\ldots$ & $\ldots$ & 1800 \\
\hline e106.wbb6_11 ......... & $\ldots$ & 2003 Nov 20.14 & Clay & Unk & 0.321 & & $\ldots$ & $\ldots$ & $\ldots$ & 19.4 & $3 \times 1200$ \\
\hline e108.wdd8_4 ........... & $2003 \mathrm{~km}$ & 2003 Nov 20.21 & Clay & Ia & $\ldots$ & 0.47 & -8 & $90 \mathrm{~N}$ & Good & 21.8 & $3 \times 1200$ \\
\hline e108.wdd8_4 …........ & $2003 \mathrm{~km}$ & 2003 Nov 21.21 & VLT & Ia & $\ldots$ & 0.47 & -8 & $90 \mathrm{~N}$ & Good & 21.8 & $2 \times 1800$ \\
\hline e118.waa5_11 .......... & $\ldots$ & 2003 Nov 22.03 & VLT & AGN & 0.556 & $\ldots$ & $\ldots$ & $\ldots$ & $\ldots$ & $\ldots$ & $2 \times 1200$ \\
\hline e119.wbb1_7 …........ & $\ldots$ & 2003 Nov 23.19 & VLT & Gal & 0.560 & $\ldots$ & $\ldots$ & $\ldots$ & $\ldots$ & $\ldots$ & 1800 \\
\hline e120.waa5_9............ & $\ldots$ & 2003 Nov 22.05 & Clay & Gal & 0.298 & $\ldots$ & $\ldots$ & $\ldots$ & $\ldots$ & $\ldots$ & 1200 \\
\hline e132.wcc1_7 ............... & $2003 \mathrm{kn}$ & 2003 Nov 22.08 & VLT & Ia & 0.244 & 0.24 & -6 & $90 \mathrm{~N}$ & Good & 21.3 & $2 \times 1800$ \\
\hline e133.wcc1_7 ............ & $\ldots$ & 2003 Nov 22.08 & VLT & Gal & 0.244 & $\ldots$ & $\ldots$ & $\ldots$ & $\ldots$ & $\ldots$ & $2 \times 1800$ \\
\hline e136.wcc1_12 .......... & 2003ko & 2003 Nov 22.13 & VLT & Ia & 0.360 & 0.36 & -11 & $94 \mathrm{D}$ & Good & 21.7 & $2 \times 1800$ \\
\hline e138.wdd4_1 ........... & $2003 \mathrm{kt}$ & 2003 Nov 23.10 & VLT & Ia & $\ldots$ & 0.61 & +5 & $95 \mathrm{D}$ & Good & 22.8 & $3 \times 1200$ \\
\hline e140.wdd5_15 ......... & $2003 \mathrm{kq}$ & 2003 Nov 22.29 & VLT & Ia & 0.606 & 0.62 & -8 & $90 \mathrm{~N}$ & Good & 22.6 & $3 \times 1200$ \\
\hline e141.wdd7_2 …........ & $\ldots$ & 2003 Nov 22.16 & Clay & II & 0.099 & $\ldots$ & $\ldots$ & $\ldots$ & $\ldots$ & $\ldots$ & $2 \times 1200$ \\
\hline e143.wdd7_3 ........... & $\ldots$ & 2003 Nov 23.15 & VLT & Unk & $\ldots$ & & $\ldots$ & $\ldots$ & $\ldots$ & $\ldots$ & $2 \times 1200$ \\
\hline e147.wdd5_9 ........... & $2003 \mathrm{kp}$ & 2003 Nov 22.18 & VLT & Ia & $\ldots$ & 0.64 & -7 & $89 \mathrm{~B}$ & Good & 22.1 & $2 \times 1800$ \\
\hline e148.wdd5_10 .......... & $2003 \mathrm{kr}$ & 2003 Nov 22.23 & VLT & Ia & 0.427 & 0.42 & -7 & $90 \mathrm{~N}$ & Good & 22.0 & $3 \times 1200$ \\
\hline e149.wdd5_10 ......... & $2003 \mathrm{ks}$ & 2003 Nov 23.28 & VLT & Ia? & $\ldots$ & 0.51 & +12 & $95 \mathrm{bd}$ & Poor & 22.2 & $3 \times 1200$ \\
\hline e309.waa9_14.......... & $\ldots$ & 2003 Nov 23.32 & GMOS & M star & $\ldots$ & $\ldots$ & $\ldots$ & $\ldots$ & $\ldots$ & $\ldots$ & $3 \times 1200$ \\
\hline e315.wbb9_3 ............. & $2003 \mathrm{ku}$ & 2003 Nov 24.31 & GMOS & Ia? & $\ldots$ & 0.79 & +0 & $94 \mathrm{~S}$ & Poor & 22.9 & $3 \times 1200$ \\
\hline e418.wcc2_8 ............. & $\ldots$ & 2003 Nov 27 & Baade & N.S. & $\ldots$ & $\ldots$ & $\cdots$ & $\ldots$ & $\ldots$ & $\ldots$ & $\ldots$ \\
\hline e501.waa1_1 ............. & $\ldots$ & 2003 Nov 28 & Baade & N.S. & $\ldots$ & $\ldots$ & $\ldots$ & $\ldots$ & $\ldots$ & $\ldots$ & $\ldots$ \\
\hline e504.waa3_4 ............ & $\ldots$ & 2003 Nov 29.05 & Baade & AGN & 0.674 & $\ldots$ & $\ldots$ & $\ldots$ & $\ldots$ & 23.2 & $3 \times 1800$ \\
\hline e510.waa1_13 .......... & $\ldots$ & 2003 Nov 29.08 & GMOS & Unk & $\ldots$ & $\ldots$ & $\ldots$ & $\ldots$ & $\ldots$ & 23.0 & 1800 \\
\hline e528.wcc5_3 ............... & $\ldots$ & 2003 Nov 28 & Baade & N.S. & $\ldots$ & $\ldots$ & $\ldots$ & $\ldots$ & $\ldots$ & 23.4 & $\ldots$ \\
\hline e529.wcc5_3 ............ & $\ldots$ & 2003 Nov 29.10 & Clay & Unk & $\ldots$ & $\ldots$ & $\ldots$ & $\ldots$ & $\ldots$ & 23.8 & $3 \times 1800$ \\
\hline e531.wcc1_4............ & $2003 \mathrm{kv}$ & 2003 Nov 29.14 & Baade & Ia? & $\ldots$ & 0.78 & -3 & $95 \mathrm{E}$ & Poor & 23.4 & $3 \times 1800$ \\
\hline f001.wbb7_1 ........... & 20031g & 2003 Dec 19.17 & MMT & Unk & $\ldots$ & $\ldots$ & $\ldots$ & $\ldots$ & $\ldots$ & 22.5 & $3 \times 1800$ \\
\hline f011.wcc7_12 .......... & $2003 \mathrm{lh}$ & 2003 Dec 21.31 & KI/LRIS & $\mathrm{Ia}$ & $\ldots$ & 0.54 & +4 & $90 \mathrm{~N}$ & Good & 22.7 & 1500 \\
\hline f017.wbb9_10 ......... & $\ldots$ & 2003 Dec 20.33 & GMOS & AGN & 0.725 & $\ldots$ & $\ldots$ & $\ldots$ & $\ldots$ & 22.7 & $3 \times 1200$ \\
\hline f041.wbb6_8 ............ & 2003le & 2003 Dec 20.23 & KI/LRIS & Ia & $\ldots$ & 0.56 & -4 & 94D & Good & 22.7 & $2 \times 1200$ \\
\hline f044.wbb8_8 ............ & $\ldots$ & 2003 Dec 21.10 & MMT & Gal & 0.409 & $\ldots$ & $\ldots$ & $\ldots$ & $\ldots$ & $\ldots$ & $3 \times 1800$ \\
\hline f076.wbb9_01 .. & $20031 f$ & 2003 Dec 21.29 & KI/LRIS & Ia & & 0.41 & +3 & $94 \mathrm{ae}$ & Good & 22.1 & 900 \\
\hline
\end{tabular}


TABLE 2-Continued

\begin{tabular}{|c|c|c|c|c|c|c|c|c|c|c|c|}
\hline ESSENCE ID ${ }^{\mathrm{a}}$ & IAUC ID $^{\mathrm{b}}$ & UT Date $^{c}$ & Telescope & Type $^{\mathrm{d}}$ & $\begin{array}{c}z \\
(\mathrm{Gal} .)^{\mathrm{e}}\end{array}$ & $\begin{array}{c}z \\
(\mathrm{SNID})^{\mathrm{f}}\end{array}$ & $\begin{array}{c}\text { Epoch } \\
{\text { (SNID })^{\mathrm{g}}}^{\text {(S) }}\end{array}$ & Template $^{\mathrm{h}}$ & Grade $^{i}$ & $\begin{array}{l}\text { Discovery }{ }^{\mathrm{j}} \\
\text { (mag) }\end{array}$ & $\begin{array}{l}\text { Exp. } \\
\text { (s) }\end{array}$ \\
\hline f076.wbb9_1 ............ & $20031 f$ & 2003 Dec 21.17 & MMT & Ia & $\ldots$ & 0.41 & +4 & $95 \mathrm{D}$ & Good & 22.1 & $3 \times 900$ \\
\hline f095.wcc2_8 ............ & & 2003 Dec 20.31 & KI/LRIS & Gal & 0.313 & & .. & & & 21.6 & $3 \times 1200$ \\
\hline f096.waa3_3 ............ & $20031 \mathrm{~m}$ & 2003 Dec 21.28 & KI/LRIS & Ia & 0.408 & 0.41 & -1 & $92 \mathrm{~A}$ & Good & 22.5 & 1500 \\
\hline f116.wbb1_7............... & $\ldots$ & 2003 Dec 20 & KI/LRIS & N.S. & $\ldots$ & $\ldots$ & $\ldots$ & $\ldots$ & $\ldots$ & 21.6 & $\ldots$ \\
\hline f123.wcc1_7 ............ & $\ldots$ & 2003 Dec 21.34 & KI/LRIS & Gal & 0.526 & $\ldots$ & $\ldots$ & $\ldots$ & $\ldots$ & 21.6 & $2 \times 1200$ \\
\hline f213.wbb4_12 ......... & $\ldots$ & 2003 Dec 19.34 & GMOS & Unk & $\ldots$ & $\ldots$ & $\ldots$ & $\ldots$ & $\ldots$ & $\ldots$ & $2 \times 1200$ \\
\hline f216.wdd4_15 .......... & 200311 & 2003 Dec 21.44 & KI/LRIS & Ia & 0.596 & 0.60 & +5 & $95 \mathrm{E}$ & Good & 21.6 & 1800 \\
\hline f221.wcc4_14 .......... & $20031 \mathrm{k}$ & 2003 Dec 21.36 & KI/LRIS & Ia? & 0.442 & 0.45 & +2 & $94 \mathrm{ae}$ & Poor & 22.8 & 1500 \\
\hline f231.waal_13 ........... & $2003 \ln$ & 2003 Dec 21.25 & KI/LRIS & Ia & $\ldots$ & 0.63 & +0 & $81 \mathrm{~B}$ & Good & 22.9 & 1500 \\
\hline f235.wbb5_13 .......... & $20031 j$ & 2003 Dec 20.27 & KI/LRIS & Ia & 0.417 & 0.42 & +5 & $95 \mathrm{D}$ & Good & 22.1 & $2 \times 1200$ \\
\hline f244.wdd3_8 ............ & $20031 \mathrm{i}$ & 2003 Dec 20.42 & KI/LRIS & Ia & 0.544 & 0.54 & -1 & $95 \mathrm{E}$ & Poor & 22.8 & $2 \times 1800$ \\
\hline 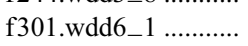 & $\ldots$ & 2003 Dec 21.42 & KI/LRIS & Ia? & $\ldots$ & 0.52 & -3 & $86 \mathrm{G}$ & Good & 21.6 & 1500 \\
\hline f304.wdd6_2 ............ & $\ldots$ & 2003 Dec 21.39 & KI/LRIS & Unk & $\ldots$ & $\ldots$ & $\ldots$ & $\ldots$ & $\ldots$ & 21.6 & 1800 \\
\hline f308.wdd6_10 .......... & $\ldots$ & 2003 Dec 20.37 & KI/LRIS & Ia? & $\ldots$ & 0.39 & -7 & 94D & Good & 21.6 & $3 \times 1800$ \\
\hline f441.wbb6_7 ............ & $\ldots$ & 2003 Dec 23.25 & GMOS & Unk & $\ldots$ & $\ldots$ & $\ldots$ & $\ldots$ & $\ldots$ & $\ldots$ & $3 \times 1200$ \\
\hline
\end{tabular}

a ESSENCE internal identification. The first letter indicates the month in the observing season. This is followed by a sequential number as targets are discovered.

The remaining letters and numbers show the specific ESSENCE field in which the object was located (R. C. Smith et al. 2005, in preparation).

${ }^{b}$ Note that not all objects judged to be SNe have official IAU names.

c The UT date at the midpoint of the observation(s).

d Our best guess as to classification of the object. Ia? indicates a lack of certainty in the identification as an SN Ia. II? indicates a lack of certainty in the identification as an SN II. Objects marked Unk are unknown. N.S. indicates that the telescope was pointed at the object, but no exposure was taken or the exposure contained no signal.

e Redshift measured from narrow emission or absorption lines from the host galaxy.

${ }^{\mathrm{f}}$ Redshift measured from the SN spectrum by SNID.

${ }^{g}$ Age of the SN relative to maximum brightness based on comparisons with SNID templates.

h Template spectrum in SNID that provides the best match to the observed spectrum.

i The "good" SNID fits correspond to $r \times$ lap $\geq 5$ (see description in text).

j Magnitude at discovery, not at time of spectroscopy.

${ }^{\mathrm{k}}$ As a result of problems with the LRIS spectrograph, b027 was observed for $1800 \mathrm{~s}$ on the blue side only, followed by a gap of a little over half an hour; it was then observed for $1800 \mathrm{~s}$ with both the blue and red sides. The time of observation listed is halfway between the midpoints of the two observations.

${ }^{1}$ The SNID analysis was performed on the weighted combination of the GMOS and Clay spectra.

${ }^{m}$ Objects d009 and d106 are the same, inadvertently assigned two different internal identifications.

${ }^{\mathrm{n}}$ SN 1987M is of Type Ic (Filippenko et al. 1990).

${ }^{\circ}$ Objects d093 and e142 are the same, inadvertently assigned two different internal identifications.

p Objects e029 and e 121 are the same, inadvertently assigned two different internal identifications.

because of poor sky conditions or because the target was actually a solar system object and had moved out of the field.

No attempt has been made to remove host-galaxy contamination for any object presented in Figures 4-11. The amount of galaxy light is significant for some objects (e.g., f221 in Fig. 8). In addition, no extinction corrections have been applied, either for Galactic reddening or extinction in the host galaxy. Given the Galactic latitudes of the ESSENCE fields (R. C. Smith et al. 2005, in preparation) and our preference for targets well separated from the host galaxy, the effects of extinction are likely to be minimal (S. Blondin et al. 2005, in preparation; R. J. Foley et al. 2005, in preparation).

For each SN Ia in Figures 4-10, the best match low-redshift comparison spectrum as determined using SNID is included (the low-redshift SN is listed in Table 2). In general, the highredshift $\mathrm{SNe}$ Ia look very similar to those at low redshift, implying that there are no significant evolutionary effects. Future papers will deal in much greater detail with the comparison with low-redshift $\mathrm{SNe}$ Ia, as well as with removal of host-galaxy contamination and the effects of extinction (S. Blondin et al. 2005, in preparation; R. J. Foley et al. 2005, in preparation).

While most of the high-redshift SNe Ia appear to be normal, there are some examples of peculiar SNe Ia. Both b004 (SN 2002iv; Fig. 4) and d083 (SN 2003jn; Fig. 5) show strong similarities to SN 1991T, an overluminous SN Ia (Filippenko et al. 1992; Phillips et al. 1992). Given the high rate of peculiar
SNe Ia at low redshift ( $\mathrm{Li}$ et al. 2001), we would expect to find such objects in a high-redshift sample.

We note that in Figures 4-10 there are several examples of high-redshift SNe Ia from the ESSENCE sample for which the low-redshift template appears to be a poor match. Examples include d086 (2003-10-27), b008 (2002-11-06), e149, and b022 (2002-12-05). The most likely explanation for this is that the templates included in SNID do not cover the complete range of possibilities, although problems with the spectrum (e.g., poor $\mathrm{S} / \mathrm{N}$ or sky subtraction) may also play a role. Rather than perform a nonobjective search for the best match, we leave these as examples of the current limitations of SNID. We plan to expand the SNID templates to eliminate such occurrences in the future.

Two unusual cases in the sample of SNe Ia spectra are e315 (SN 2003ku) and b004 (SN 2002iv). They are the only SNe for which the redshift determination in SNID was ambiguous. For e315, if we assume that it is an SN Ia, then the best-fit redshift is 0.79 , but a fit at a redshift of 0.41 is only marginally worse. All other SN Ia spectra in the ESSENCE sample had redshifts determined by SNID that were unambiguous. If we rely on the spectrum alone, the SNID result of $z=0.79$ is what we would choose, and therefore we report it in this paper. Analysis presented by K. Krisciunas et al. (2005, in preparation) shows that neither of the redshifts suggested by SNID gives a very satisfactory fit to the photometry. In the case of b004, the SNID result is $z=0.39$, while the fit when $z=0.231$, known from 


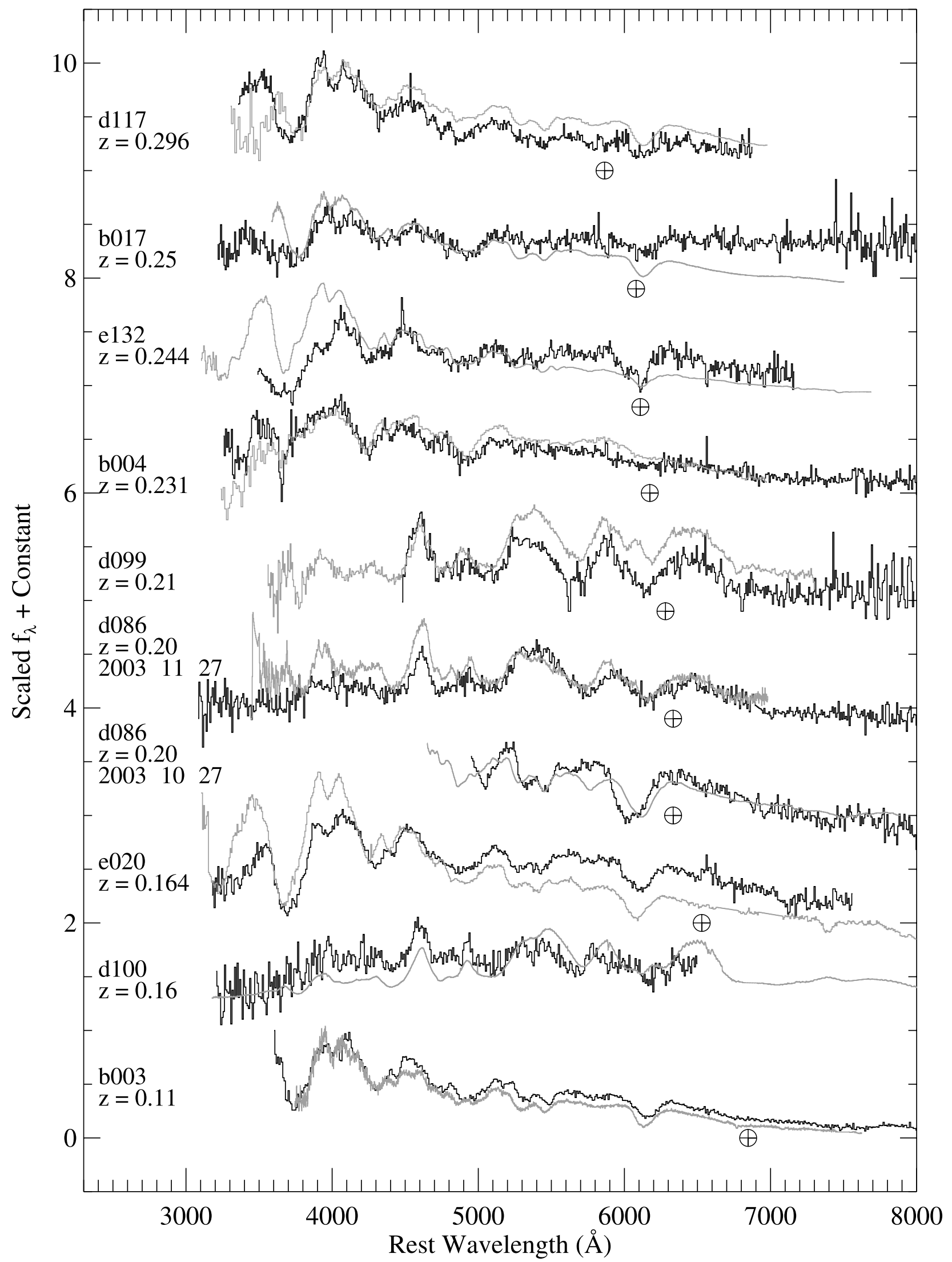

FIG. 4.- Rest-wavelength spectra of SNe Ia (or likely SNe Ia) from the first 2 years of the ESSENCE project in order of increasing redshift. Each ESSENCE SN (black line) is overplotted by a low-redshift SN Ia (gray line) for comparison. The low-redshift SN Ia used for comparison is listed in Table 2. In addition, each spectrum is labeled with the ESSENCE identification number and the deduced redshift. Spectra of the uncertain SNe Ia are indicated with an asterisk. The deredshifted location of the A-band atmospheric absorption feature is indicated with a telluric symbol. We usually, but not always, were able to remove this feature through division by the spectrum of a featureless standard star. The flux scale is $f$, with arbitrary additive offsets between the spectra. 


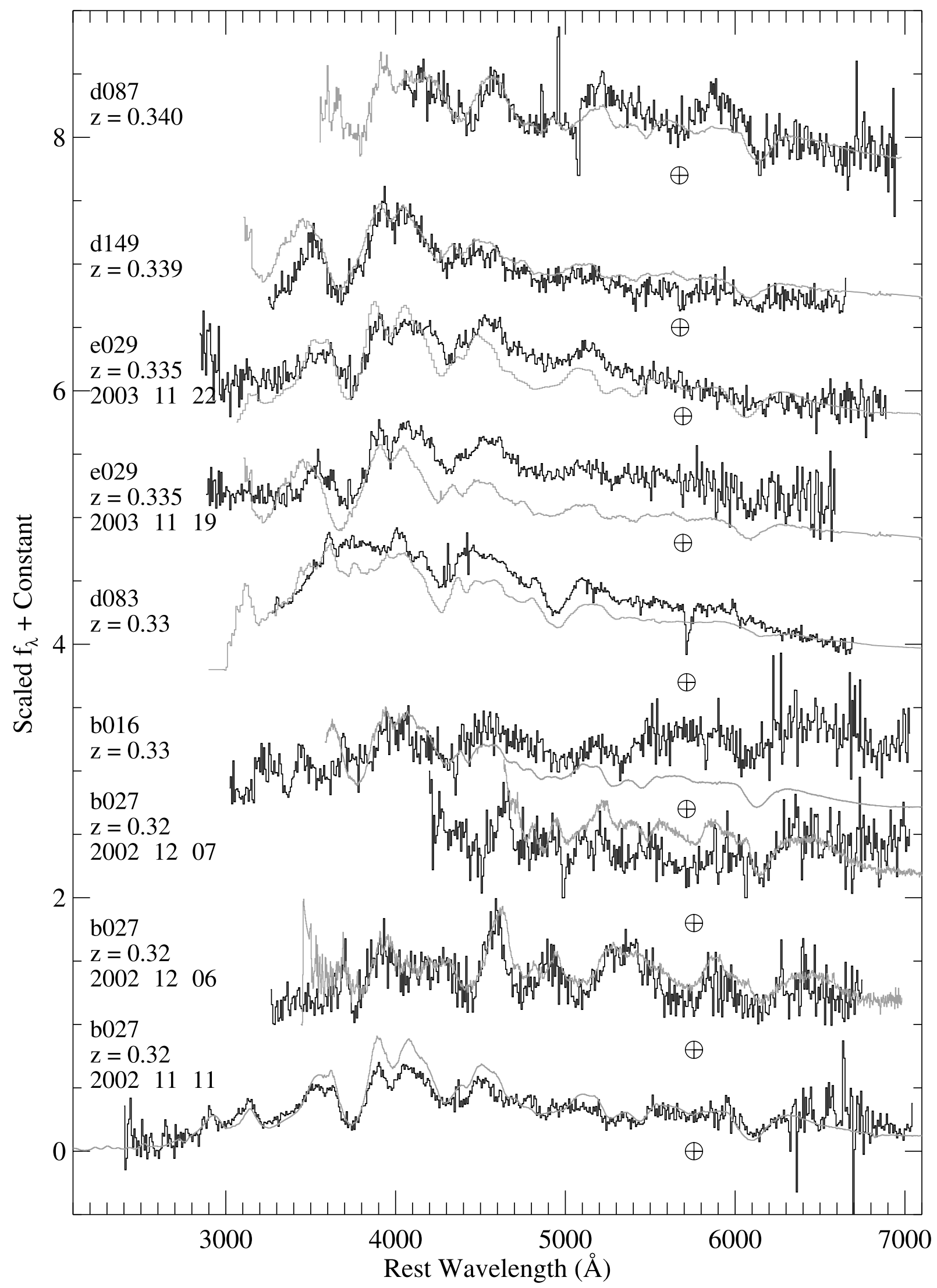

FIG. 5.-Rest-wavelength spectra of ESSENCE SNe Ia, as in Fig. 4. 


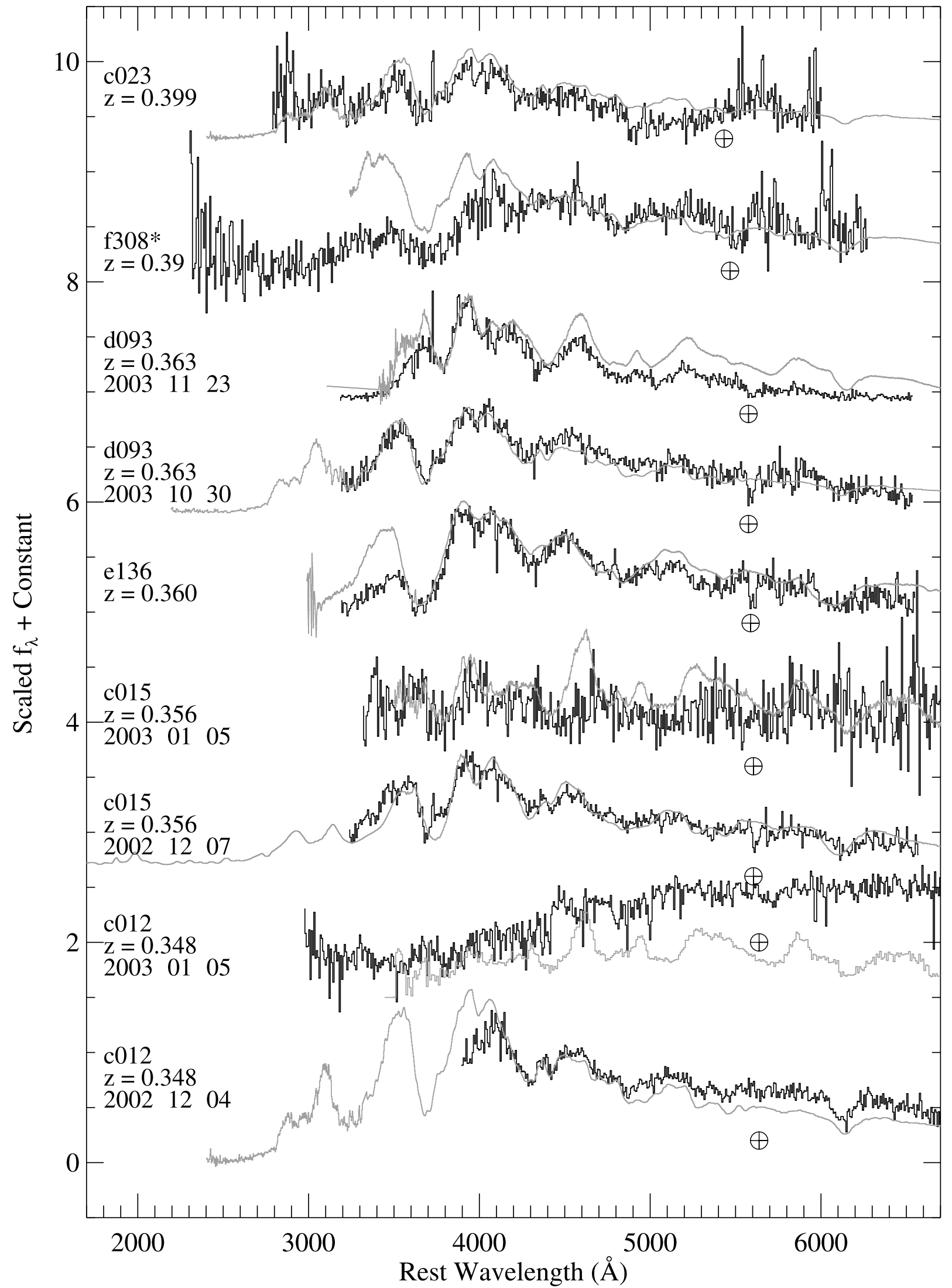

FIG. 6.-Rest-wavelength spectra of ESSENCE SNe Ia, as in Fig. 4. The 2002-12-04 spectrum of c012 is a weighted average of the Clay and GMOS spectra. 


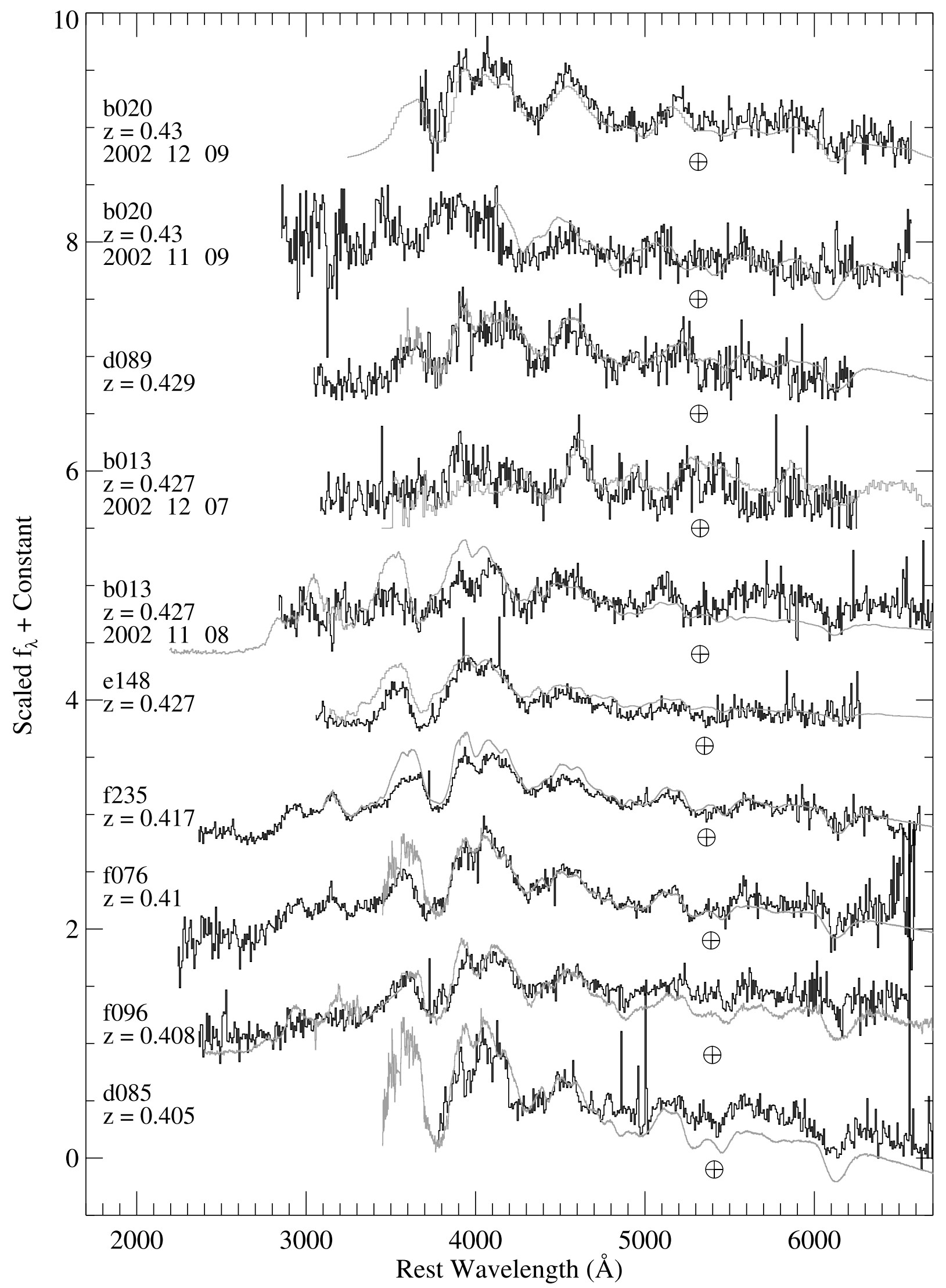

FIG. 7.- Rest-wavelength spectra of ESSENCE SNe Ia, as in Fig. 4. The spectrum of f076 is a weighted average of the MMT and Keck I LRIS spectra. 


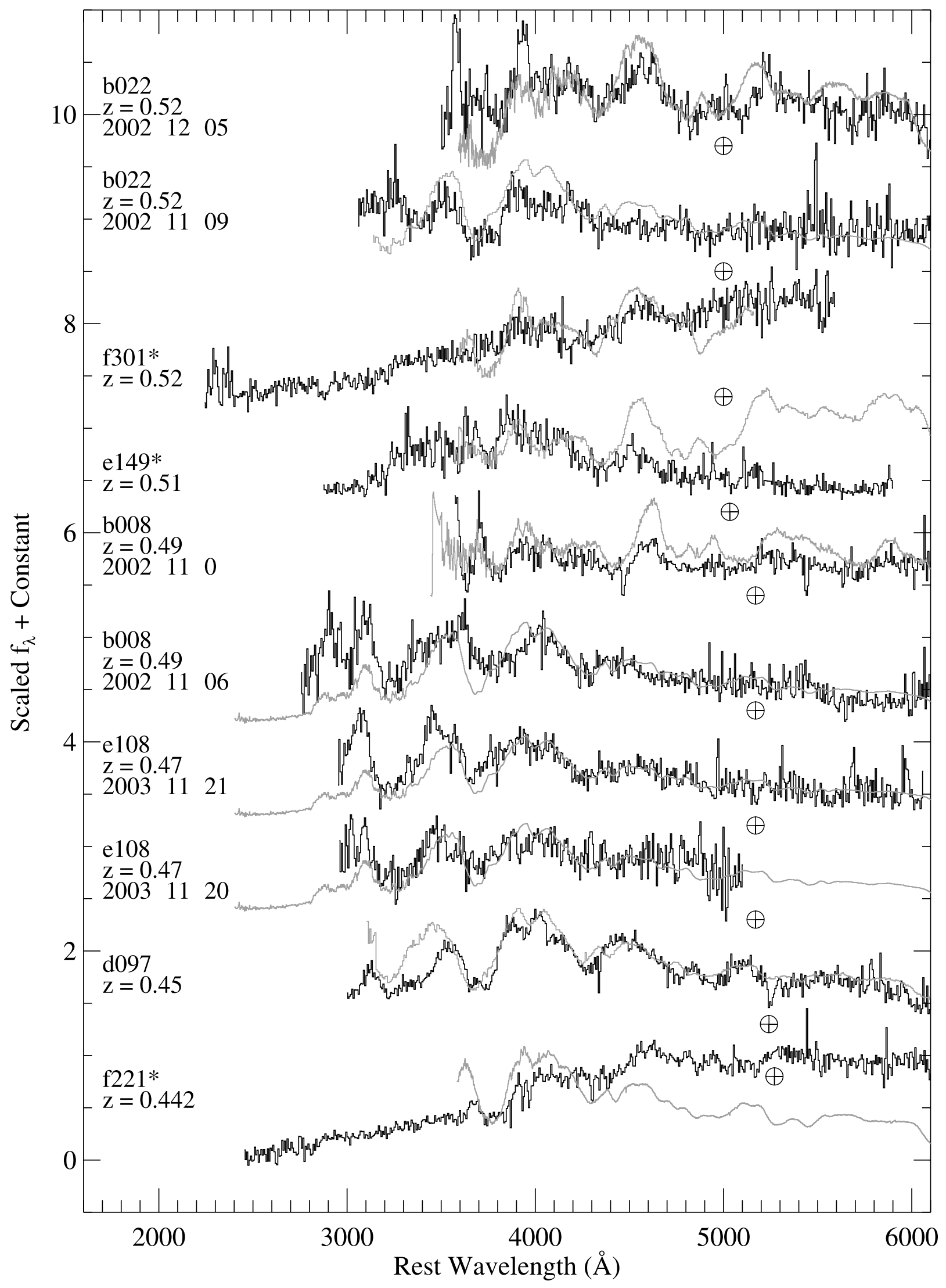

FIG. 8.-Rest-wavelength spectra of ESSENCE SNe Ia, as in Fig. 4. 


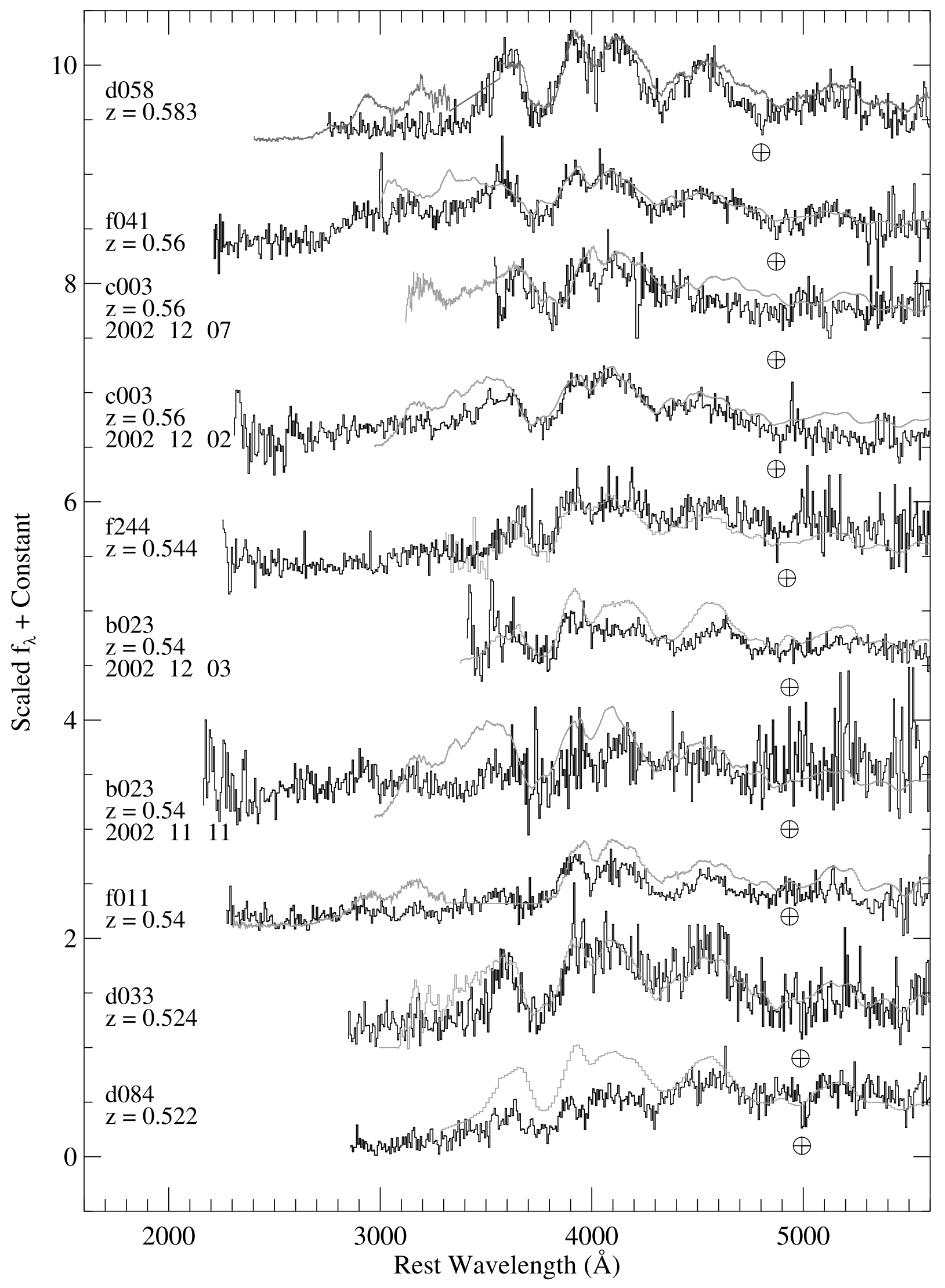

FIG. 9.-Rest-wavelength spectra of ESSENCE SNe Ia, as in Fig. 4. 


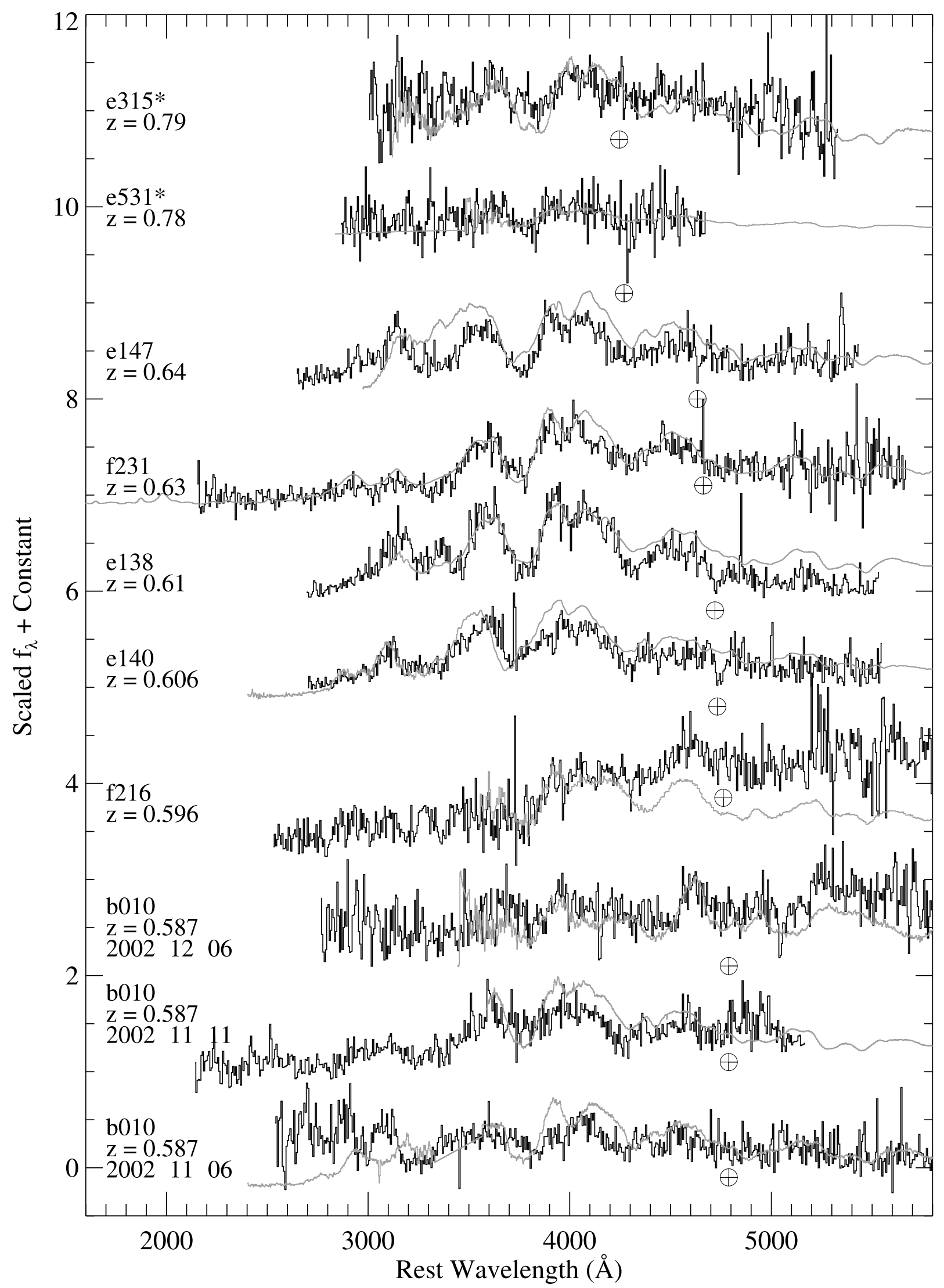

FIG. 10.- Rest-wavelength spectra of ESSENCE SNe Ia, as in Fig. 4. The GMOS and VLT spectra of b010 have been combined. 


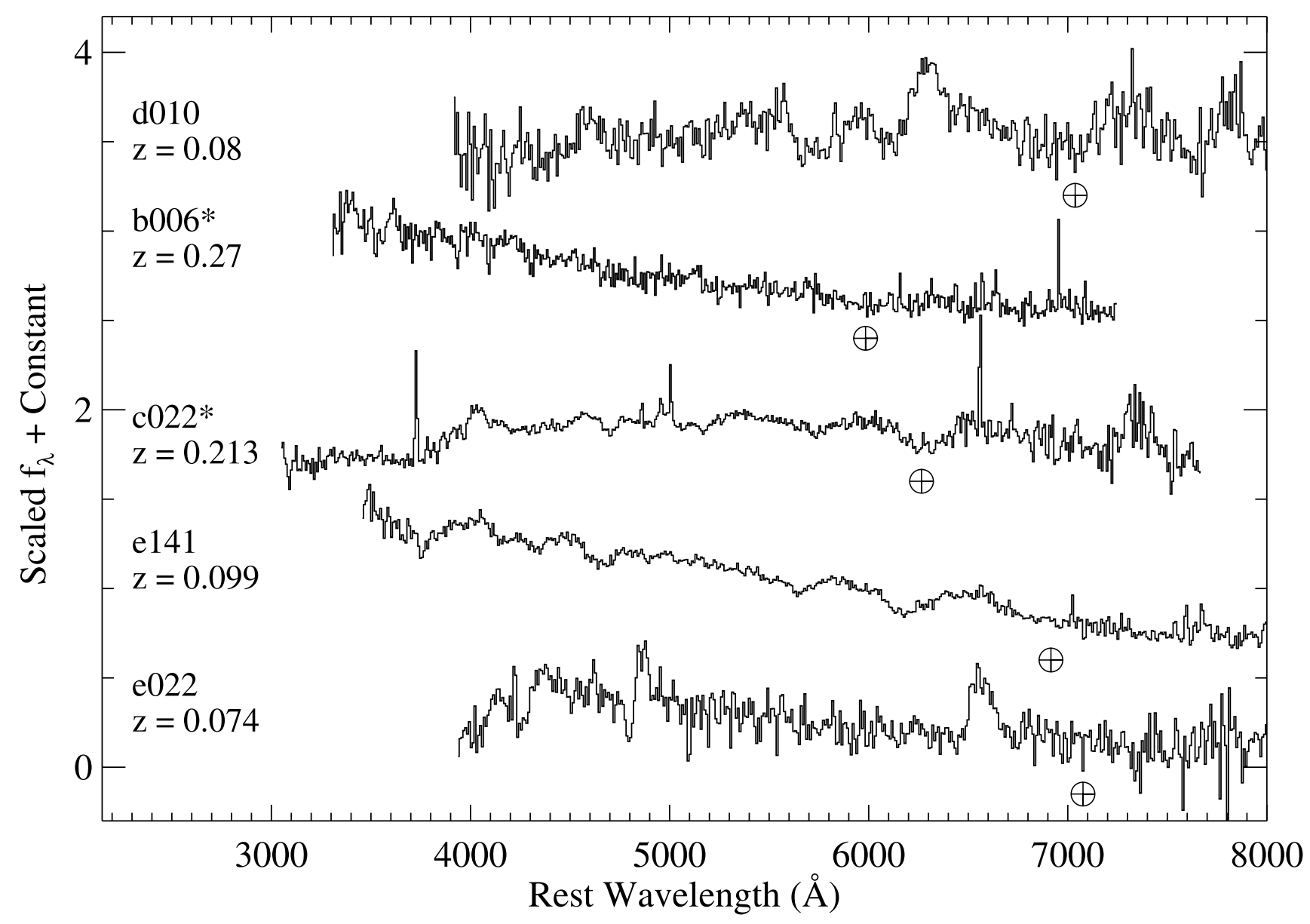

FIG. 11.- Spectra of SNe II and one SN Ib/c from the first 2 years of the ESSENCE project. Each spectrum is labeled with the ESSENCE identification number and the deduced redshift. Spectra of uncertain SNe II are indicated with an asterisk. The deredshifted location of the A-band atmospheric absorption feature is indicated with a telluric symbol. We usually, but not always, were able to remove this feature through division by the spectrum of a featureless standard star. The flux scale is $f_{\lambda}$ with arbitrary additive offsets between the spectra. The SN Ib/c is d010 (SN 2003jp). 


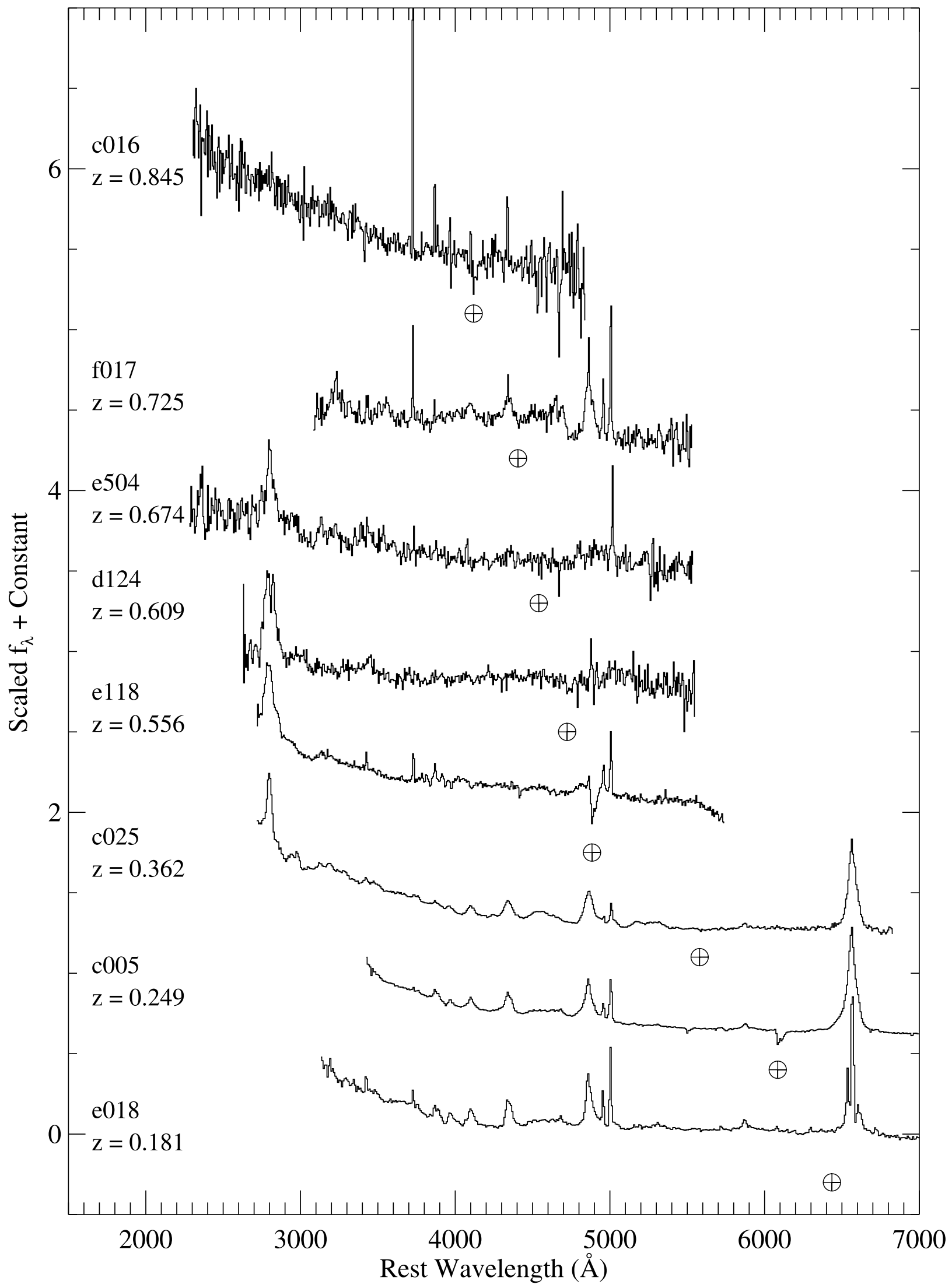

FIG. 12.- Spectra of AGNs from the first 2 years of the ESSENCE project. Each spectrum is labeled with the ESSENCE identification number and the deduced redshift. The deredshifted location of the A-band atmospheric absorption feature is indicated with a telluric symbol. We usually, but not always, were able to remove this feature through division by the spectrum of a featureless standard star. The flux scale is $f_{\lambda}$ with arbitrary additive offsets between the spectra. 


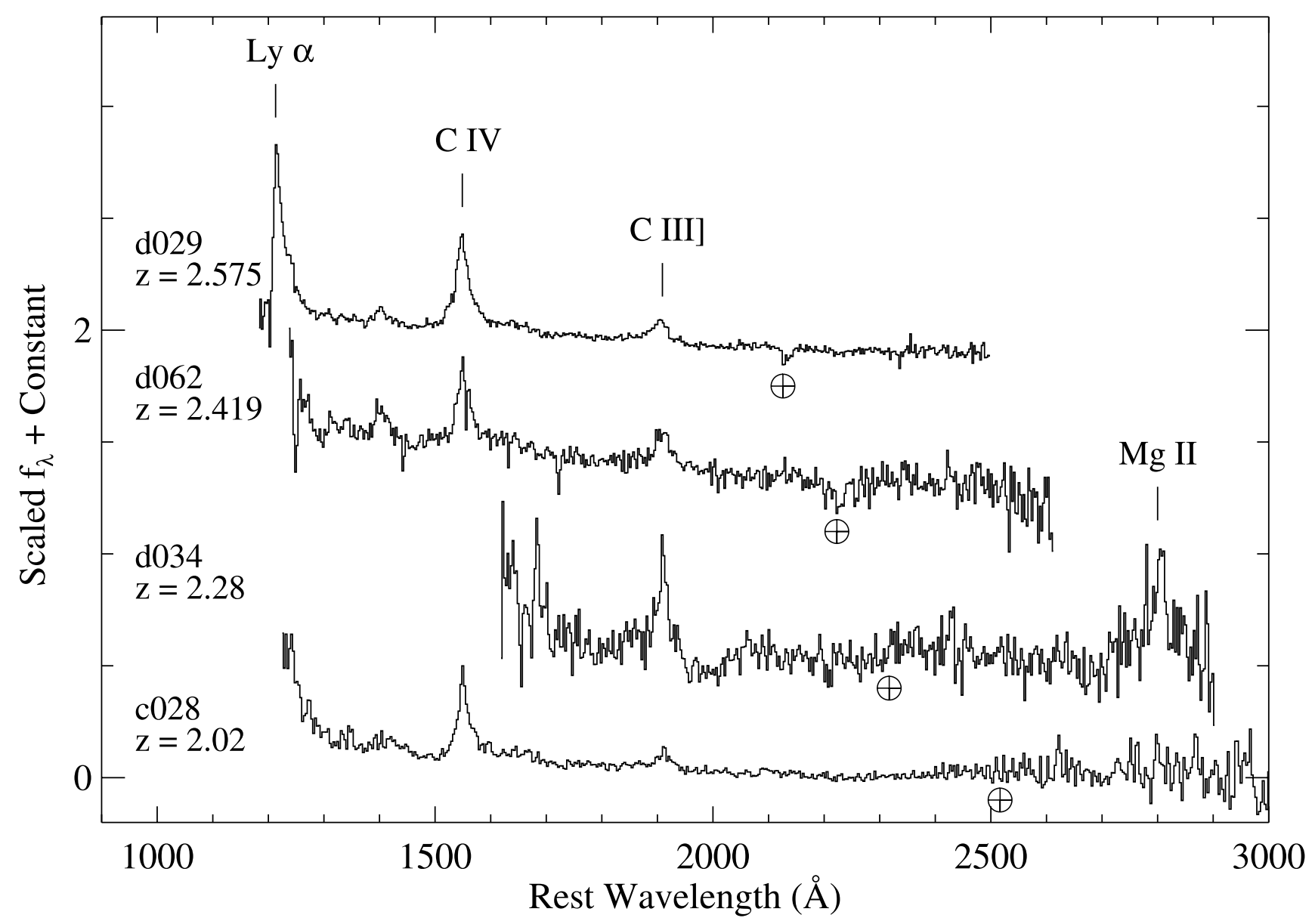

FIG. 13.- Spectra of AGNs from the first 2 years of the ESSENCE project, as in Fig. 12. 


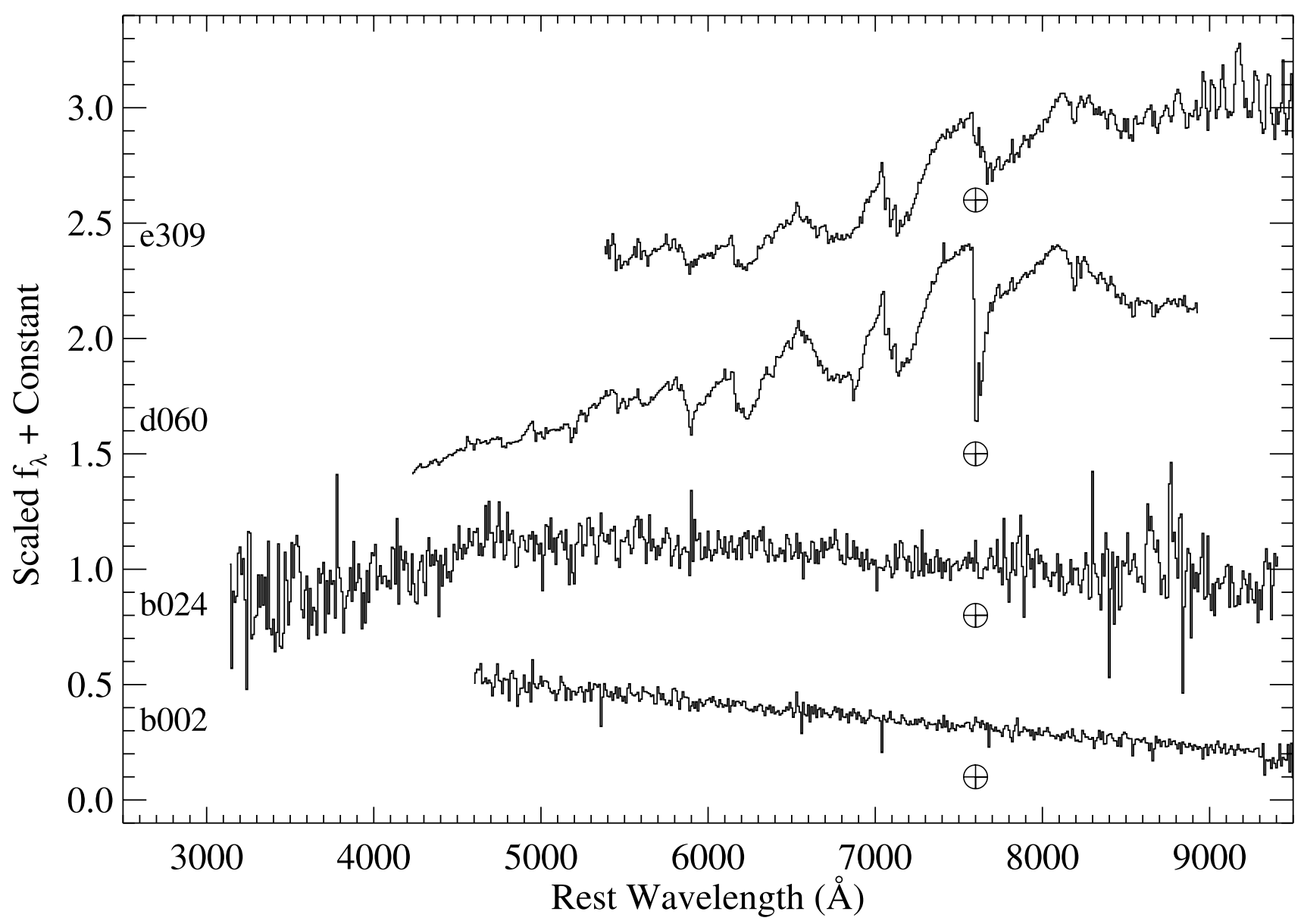

FIG. 14.- Spectra of four stars from the first 2 years of the ESSENCE project. Each spectrum is labeled with the ESSENCE identification number. The location of the A-band atmospheric absorption feature is indicated with a telluric symbol. We usually, but not always, were able to remove this feature through division by the spectrum of a featureless standard star. The flux scale is $f_{\lambda}$ with arbitrary additive offsets between the spectra. 


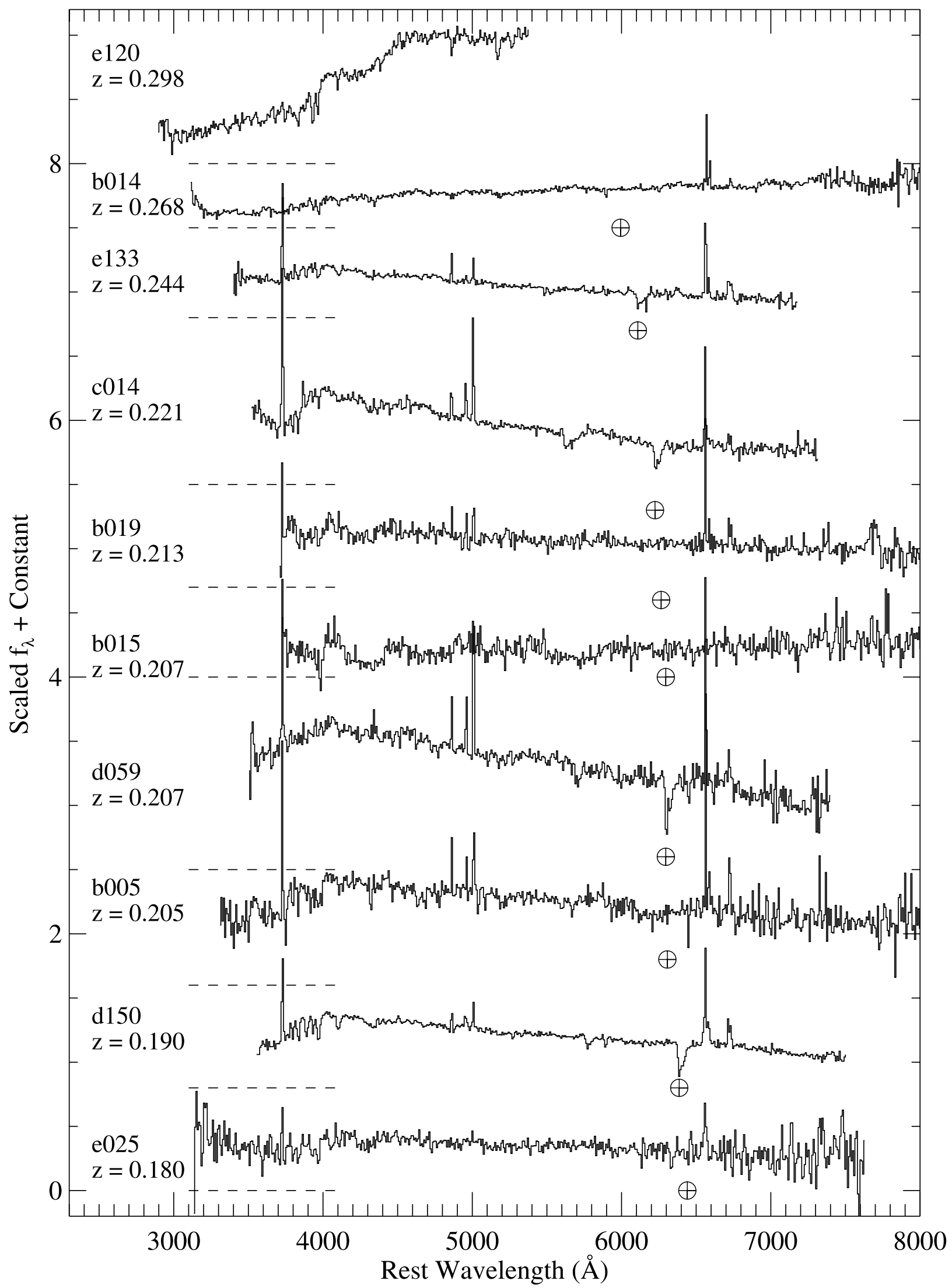

FIG. 15.- Spectra of galaxies from the first 2 years of the ESSENCE project. Each spectrum is labeled with the ESSENCE identification number and the deduced redshift. The deredshifted location of the A-band atmospheric absorption feature is indicated with a telluric symbol. We usually, but not always, were able to remove this feature through division by the spectrum of a featureless standard star. The flux scale is $f_{2}$ with arbitrary additive offsets between the spectra. The zero point of the flux scale for each spectrum is indicated (dashed line). For b005, the Keck II ESI and MMT spectra have been combined. For c014, the VLT and GMOS spectra have been combined. 


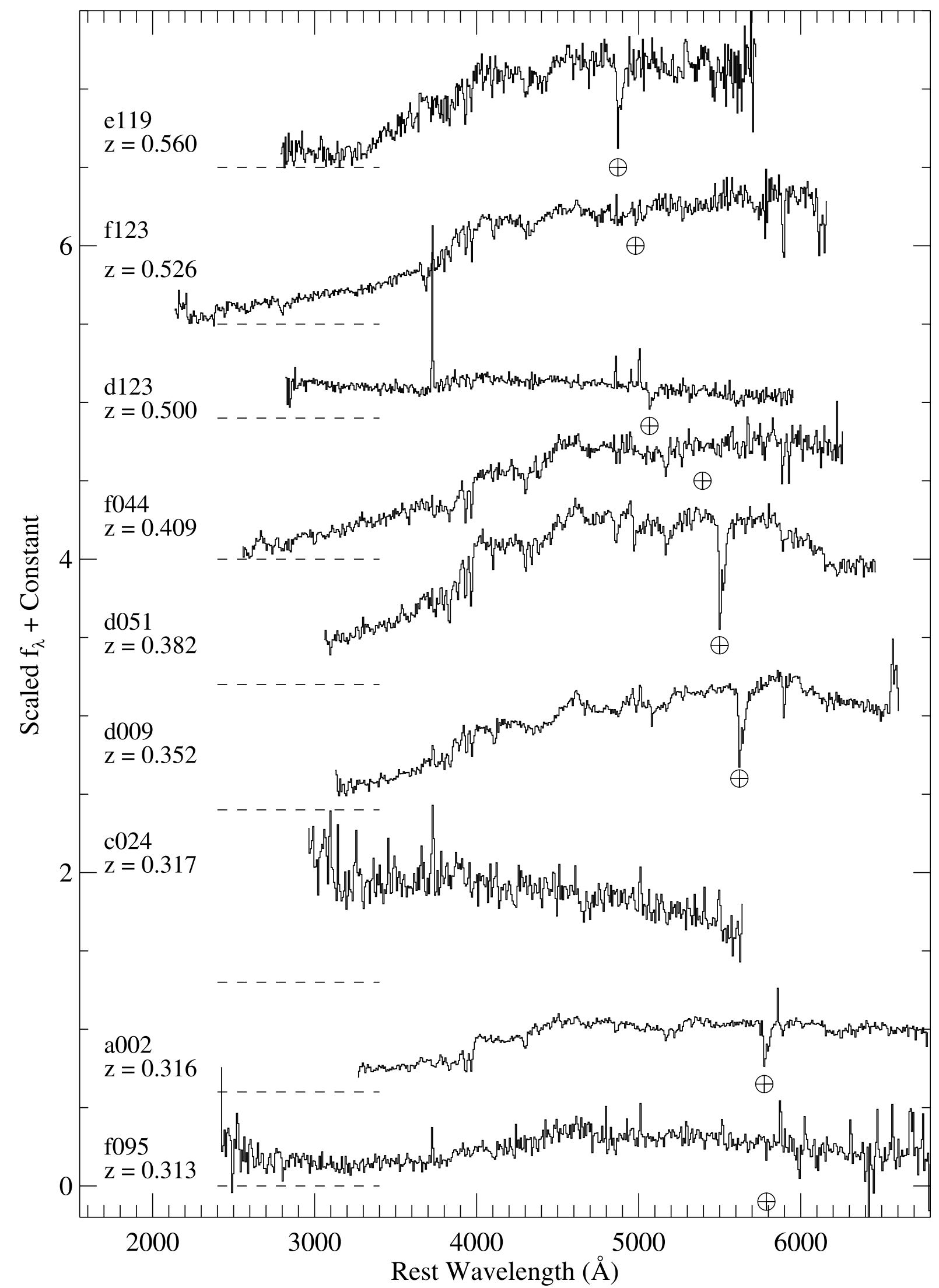

FIG. 16.- Spectra of galaxies from the first 2 years of the ESSENCE project, as in Fig. 15. For d009, the two VLT spectra have been combined. 


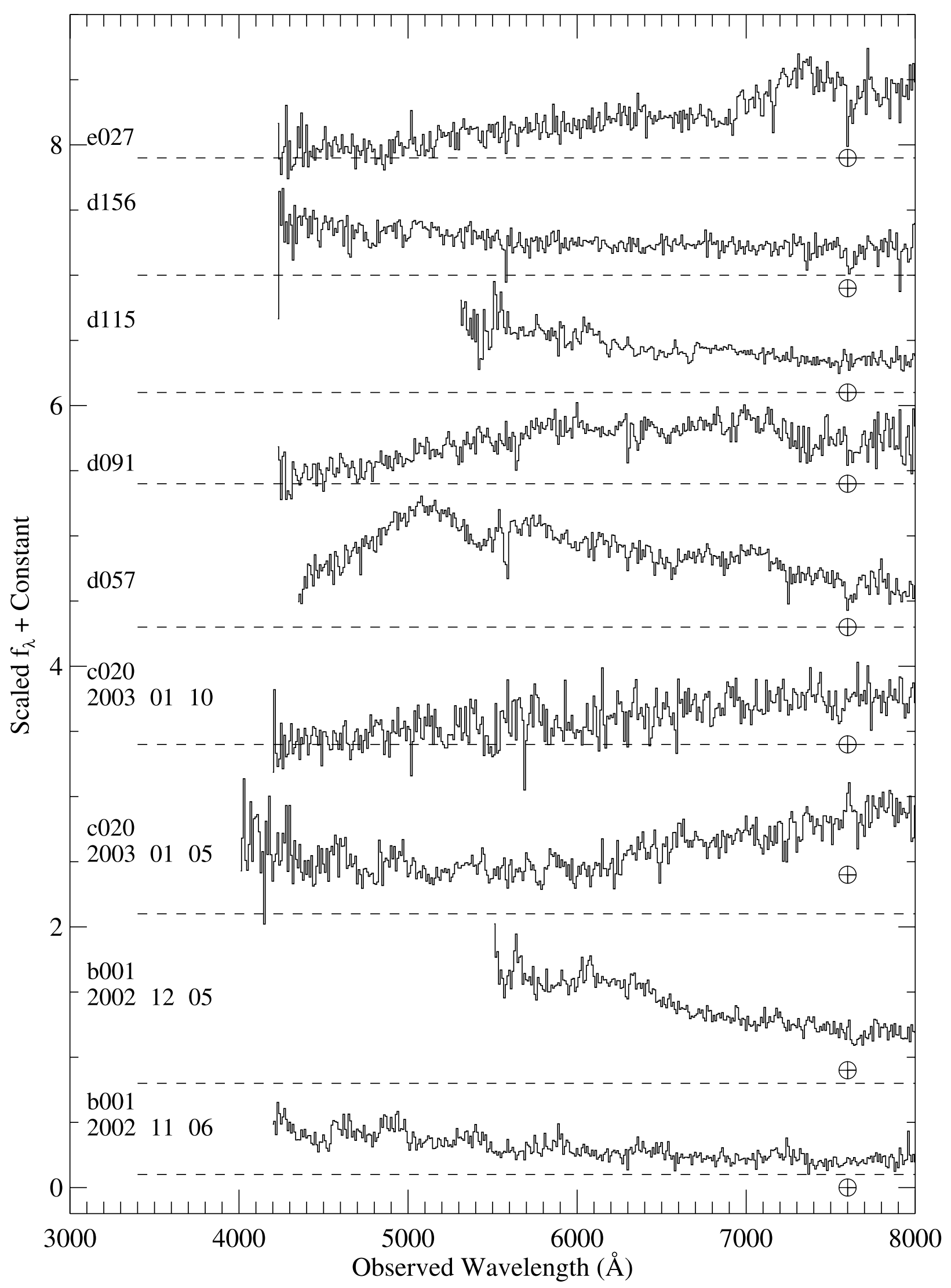

FIG. 17.-Spectra of objects whose classification is uncertain from the first 2 years of the ESSENCE project. Each spectrum is labeled with the ESSENCE identification number. The location of the A-band atmospheric absorption feature is indicated with a telluric symbol. We usually, but not always, were able to remove this feature through division by the spectrum of a featureless standard star. The flux scale is $f_{\lambda}$ with arbitrary additive offsets between the spectra. The zero point of the flux scale for each spectrum is indicated (dashed line). 


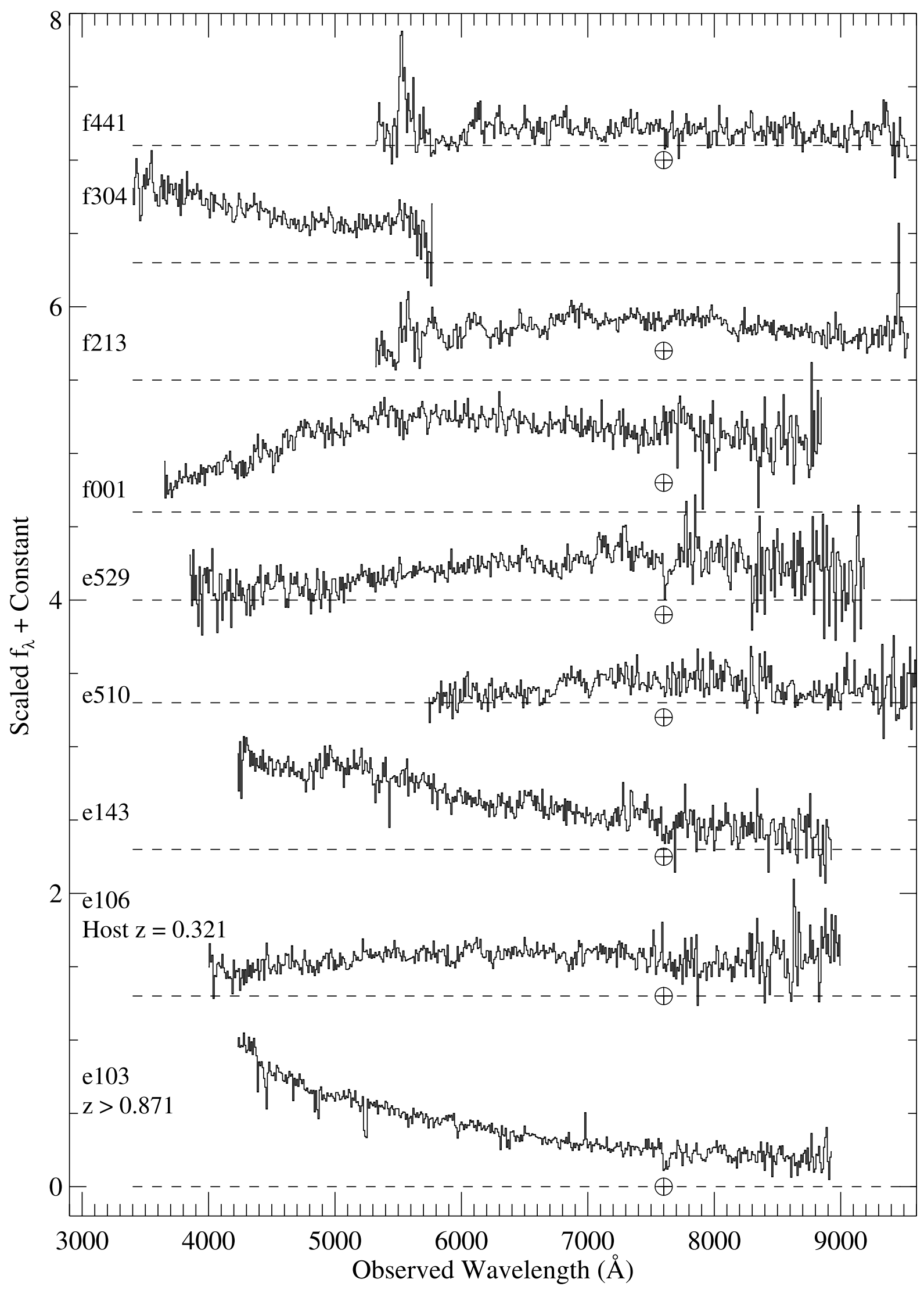

FIG. 18. - Spectra of objects whose classification is uncertain from the first 2 years of the ESSENCE project, as in Fig. 17. 
galaxy features, is almost as good. As b004 is similar to SN 1991T (see above), the lack of good templates in SNID may be the source of this discrepancy. Correlation of b004 with SN $1991 \mathrm{~T}$ templates yields a redshift of $z=0.22$, close to the value derived from the host galaxy. This highlights some of the perils of identifying optical transients with low $\mathrm{S} / \mathrm{N}$ spectra. Sometimes the spectrum alone is not enough; a consideration of all the information (spectrum, light curve, host galaxy, etc.) is necessary to draw the appropriate conclusion.

In Figure 11 we show only spectra of objects that can be definitively identified as core-collapse SNe. It is likely that some of the objects we classify as unknown (Figs. 17 and 18) are also core-collapse events. Early spectra of core-collapse SNe often show featureless blue continua, but such a spectrum could result from some other kind of object, such as an AGN. Without a redshift or identifiable line features, we could not arbitrarily classify all objects with blue continua as core-collapse SNe. There are fewer core-collapse events overall (compared to $\mathrm{SNe}$ Ia) as a result of the color selection described above (avoiding relatively blue objects) and the fact that the ESSENCE survey is magnitude limited, discriminating against the intrinsically fainter corecollapse SNe.

Among the unknown spectra (Figs. 17 and 18), there are three that require some discussion. For e106, the redshift is known because the host galaxy was also observed. The emission line appearing at the observed wavelength of $9457 \AA$ in $\mathrm{f} 213$ is real. If this is $\mathrm{H} \alpha$, then $z=0.44$; if it is [O III] $\lambda 5007$, then $z=0.89$. There is an apparent doublet absorption line in e103 at an observed wavelength of $5240 \AA$. We interpret this as $\mathrm{Mg}$ II $\lambda 2800$ at a redshift of 0.871 , implying that this object has a redshift at least that high. It is likely to be a high-redshift AGN, but we do not have enough information to move it out of the category of unknown objects.

\section{CONCLUSIONS}

We have presented optical spectroscopy of the targets selected for follow-up observations from the first 2 years of the ESSENCE project. As the target selection process has improved, we have increased our yield of SNe Ia that are needed for the primary purpose of the ESSENCE project: measuring luminosity distances to $\sim 200 \mathrm{SNe}$ Ia over the redshift range $(0.2 \lesssim z \lesssim 0.8)$. The SNe Ia show strong similarities with low-redshift SNe Ia, implying that there are no significant evolutionary changes in the nature of SNe Ia and that our methods for identifying objects have been successful. This is also shown by the concordance of redshifts derived from SN spectra and those found from the host galaxy itself. Over the next 3 years, ESSENCE will continue to discover high-redshift SNe Ia. With enough spectroscopic telescope time, we plan to be even more successful in correctly identifying SNe Ia than we have been during the first 2 years.

We would like to thank the staffs of the Paranal, Gemini, Keck, Las Campanas, MMT, F. L. Whipple, and Cerro Tololo Inter-American Observatories for their extensive assistance and support during this project. We would also like to thank Warren Brown and Craig Heinke for assistance with the MMT observations. This work is supported primarily by NSF grants AST 0206329 and AST 04-43378 and by NASA grant GO-9860 from the Space Telescope Science Institute. In addition, A. V. F. is grateful for the support of NSF grant AST 03-07894 and for a Miller Research Professorship at the University of California, Berkeley, during which part of this work was completed. C. W. S. thanks the McDonnell Foundation and Harvard University for their support. A. C. acknowledges the support of CONICYT (Chile) through FONDECYT grants 1000524 and 7000524.
Appenzeller, I., et al. 1998, Messenger, 94, 1

Barris, B. J., \& Tonry, J. L. 2004, ApJ, 613, L21

Barris, B. J., et al. 2004, ApJ, 602, 571

Blondin, S., Walsh, J. R., Leibundgut, B., \& Sainton, G. 2005, A\&A, 431, 757

Challis, P. 2002, IAU Circ., 8034, 2 2003, IAU Circ., 8251,

Coil, A. L., et al. 2000, ApJ, 544, L111

Covarrubias, R. 2003, IAU Circ., 8238, 1

Covarrubias, R., Alda, A., Prieto, J. L., Berlind, P., Blondin, S., Leibundgut, B., \& Matheson, T. 2003, IAU Circ., 8237, 2

Dressler, A. 2004, A User's Manual for IMACS (Pasadena: Carnegie Obs.), http://www.ociw.edu/lco/magellan/instruments/IMACS/observing_with_ IMACS_2.html

Fabricant, D., Cheimets, P., Caldwell, N., \& Geary, J. 1998, PASP, 110, 79

Filippenko, A. V. 1982, PASP, 94, 715

1997, ARA\&A, 35, 309

2004, in Measuring and Modeling the Universe, ed. W. L. Freedman

(Cambridge: Cambridge Univ. Press), 270

. 2005, in White Dwarfs: Probes of Galactic Structure and Cosmology,

ed. E. M. Sion, H. L. Shipman, \& S. Vennes (Dordrecht: Kluwer), in press (astro-ph/0410609)

Filippenko, A. V., Porter, A. C., \& Sargent, W. L. W. 1990, AJ, 100, 1575

Filippenko, A. V., et al. 1992, ApJ, 384, L15

Gal-Yam, A., Poznanski, D., Maoz, D., Filippenko, A. V., \& Foley, R. J. 2004, PASP, 116, 597

Garnavich, P. M., et al. 1998, ApJ, 509, 74

Glazebrook, K., \& Bland-Hawthorn, J. 2001, PASP, 113, 197

Hicken, M. 2004, IAU Circ., 8261, 1

Hook, I., et al. 2003, Proc. SPIE, 4841, 1645

Horne, K. 1986, PASP, 98, 609

Jeffery, D. J., Leibundgut, B., Kirshner, R. P., Benetti, S., Branch, D., \& Sonneborn, G. 1992, ApJ, 397, 304

Kirshner, R. P., et al. 1993, ApJ, 415, 589

\section{REFERENCES}

Knop, R. A., et al. 2003, ApJ, 598, 102

Leibundgut, B., \& Sollerman, J. 2001, Europhys. News, 32, 121

Li, W., Filippenko, A. V., Treffers, R. R., Riess, A. G., Hu, J., \& Qiu, Y. 2001, ApJ, 546, 734

Lidman, C., et al. 2005, A\&A, 430, 843

Matheson, T., Filippenko, A. V., Ho, L. C., Barth, A. J., \& Leonard, D. C. 2000, AJ, 120, 1499

Mazzali, P. A., Lucy, L. B., Danziger, I. J., Gouiffes, C., Cappellaro, E., \& Turatto, M. 1993, A\&A, 269, 423

Mulchaey, J. 2001, LDSS-2 User's Guide (Pasadena: Carnegie Obs.), http:// www.ociw.edu/lco/magellan/instruments/LDSS2/ldss2_usersguide.html

Oke, J. B., et al. 1995, PASP, 107, 375

Perlmutter, S., et al. 1998, Nature, 391, 51 1999, ApJ, 517, 565

Phillips, M. M., Wells, L. A., Suntzeff, N. B., Hamuy, M., Leibundgut, B., Kirshner, R. P., \& Foltz, C. B. 1992, AJ, 103, 1632

Poznanski, D., Gal-Yam, A., Maoz, D., Filippenko, A. V., Leonard, D. C., \& Matheson, T. 2002, PASP, 114, 833

Riess, A. G., et al. 1997, AJ, 114, 722 1998, AJ, 116, 1009 2001, ApJ, 560, 49 2004a, ApJ, 600, L163 2004b, ApJ, 607, 665

Schmidt, B. P., et al. 1998, ApJ, 507, 46

Schmidt, G., Weymann, R., \& Foltz, C. 1989, PASP, 101, 713

Sheinis, A. I., Bolte, M., Epps, H. W., Kibrick, R. I., Miller, J. S., Radovan, M. V., Bigelow, B. C., \& Sutin, B. M. 2002, PASP, 114, 851

Smith, R. C., et al. 2002, IAU Circ., 8021, 1

Strolger, L., et al. 2004, ApJ, 613, 200

Tonry, J., \& Davis, M. 1979, AJ, 84, 1511

Tonry, J. L., et al. 2003, ApJ, 594, 1

Wade, R. A., \& Horne, K. D. 1988, ApJ, 324, 411 